\title{
Hierarchical Silicoaluminophosphate Catalysts with Enhanced Hydroisomerization Selectivity by Directing the Orientated Assembly of Premanufactured Building Blocks
}

Dongliang Jin, ${ }^{\dagger}$ Guanghua Ye, ${ }^{\S}$ Jingwei Zheng, ${ }^{\dagger}$ Weimin Yang, ${ }^{\ddagger}$ Kake Zhu, ${ }^{*}{ }^{\dagger}$ Marc-Olivier Coppens, ${ }^{*},{ }^{\S}$ and Xinggui Zhou ${ }^{\dagger}$

${ }^{\dagger}$ UNILAB, State Key Lab of Chemical Engineering, School of Chemical Engineering, East China University of Science and Technology, Shanghai 200237, People's Republic of China

${ }^{\ddagger}$ Shanghai Research Institute of Petrochemical Technology, SINOPEC, Shanghai 201208, People’s Republic of China

${ }^{\S}$ Department of Chemical Engineering, University College London, London WC1E 7JE, United Kingdom

Supporting Information

ABSTRACT: The ability to generate nanoscale zeolites and direct their assembly into hierarchical structures offers a promising way to maximize their diffusion-dependent catalytic performance. Herein, we report an orientated assembly strategy to construct hierarchical architectures of silicoaluminophosphates (SAPOs) by using prefabricated nanocrystallites as a precursor. Such a synthesis is enabled by interrupting the dry gel conversion process to prepare nanocrystallites, as crystal growth is shown to proceed predominantly by particle attachment. The orientation of assembly can be controlled to form either a three-dimensional, spongelike morphology or a two-dimensional "house-of-cards" structure, by modifying the additives. Structures with a high degree of control over crystal size, shape, architecture, pore network, and acidic properties are achieved. This versatile technique avoids the more tedious and expensive templating routes that have been proposed previously. The catalytic performance for the hydroisomerization

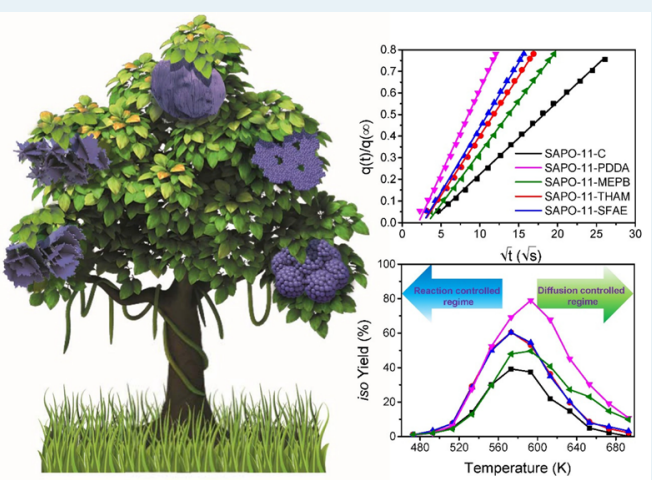
of $n$-heptane was evaluated for a series of Pt-supported catalysts, and a record isomer yield (79\%) was attained for a catalyst with spongelike architecture. The hierarchical architecture influences isomer selectivity for two reasons: expanding the intrinsicreaction-controlled regime to be able to work at higher temperatures or conversion levels, and enhancing mass transport to reduce cracking of dibranched isomers. Such an acidity-diffusivity interplay indicates that strong acidity favors isomerization operating at temperatures away from the diffusion-limited regime, while crystal size and pore connectivity are key factors for enhancing diffusion. The proposed materials offer tremendous opportunities to realize hierarchical catalyst designs that work under optimal operating conditions.

KEYWORDS: orientated assembly, hierarchical porosity, hydroisomerization, n-heptane, diffusion, silicoaluminophosphate, zeolite

\section{INTRODUCTION}

The fabrication of self-assembled, hierarchical structures of inorganic objects is an effective way to maximize the potential of processes that are dependent on structural architecture. Synthesis protocols to control the morphology, as well as the underlying controlling mechanisms, have progressed greatly over the past few years. In particular, crystallization by particle attachment (CPA) has been found to be a ubiquitous crystal nucleation and growth pathway in natural and synthetic systems. ${ }^{1}$ Since crystallization history can significantly influence the morphology of the final crystal, it is reasonable to intentionally design synthesis strategies by using this growth pathway. In particular, the formation of zeolites and zeotype crystals through attachment of various particle building blocks has been unveiled for silicalite-1 (MFI), ${ }^{2}$ analcime (ANA), ${ }^{3}$ zeolite beta (*BEA), ${ }^{4,5}$ zeolite A (LTA), ${ }^{6}$ zeolite L (LTL),
SSZ-13 chabazite (CHA), ${ }^{8}$ SAPO-5 (AFI), ${ }^{9}$ etc. The CPA growth mechanism has sparked interest in designing synthesis methods to attain structural control at the mesoscale by manipulating synthesis conditions and adding growth modifiers, ${ }^{3-5,8}$ and catalytic performance has been improved as a result. ${ }^{5}$ Nevertheless, exploring CPA growth of silicoaluminophosphates (SAPOs) is in its infancy.

SAPOs represent an important subclass of molecular sieves, along with zeolitic aluminosilicates, which are widely used as catalysts and adsorbents and for purification purposes. The observed catalytic performance of SAPOs is the result of the interplay between intrinsic catalytic activity and mass transfer.

Received: May 20, 2017

Revised: July 18, 2017

Published: July 24, 2017 
For instance, SAPO-11 is a unidimensional molecular sieve with AEL topology and elliptical pores of $4.0 \AA \times 6.5 \AA$, defined by 10 -membered rings; such a cylindrical pore structure endows it with a high monobranched $(\mathrm{MB})$ isomer selectivity in industrial catalytic long chain $\left(\mathrm{C}_{15+}\right)$ n-paraffin hydroisomerization (dewaxing) processes. Hydroisomerization occurs on a bifunctional Pt/SAPO-11 catalyst, in which olefins that were formed after dehydrogenation of $n$-paraffins on $\mathrm{Pt}$ metal sites are protonated to carbenium ion intermediates on the acidic sites of SAPO-11..$^{10-12}$ The carbenium ions undergo skeletal rearrangement on Brønsted sites to form iso-alkyl carbenium ions that can desorb as iso-alkenes with a different degree of branching or undergo $\mathrm{C}-\mathrm{C}$ bond cleavage ( $\beta$-scission) into cracking byproducts. Iso-alkenes are subsequently hydrogenated to the corresponding iso-paraffins on metal centers. As isomerization proceeds, $\mathrm{MB}$ and dibranched (DB) isomers form the major products, as further branching is restricted by the spatial constraints imposed by the micropores. ${ }^{10}$ The kinetic diameters of the isomers are increasingly larger with increasing degree of branching, leading to bulkier products; consequently, the diffusivity of such isomer products in micropores decreases and their residence time increases. ${ }^{10,13}$ Cracking rates for isomers increase with the degree of branching: indeed, lighter alkane byproducts mainly originate from cracking of DB isomers, as $n$-paraffins and $\mathrm{MB}$ isomers are not susceptible toward cracking. ${ }^{10,11,14,15}$ Consequently, the selectivity toward unwanted cracking products increases at high conversions, as a result of the long residence time of the DB isomers inside the micropores.

Recent efforts to minimize cracking in hydroisomerization have been directed to the generation of hierarchically porous SAPOs or downsizing of SAPO crystals to the nanoscale. Toward this goal, both bottom-up and top-down synthesis strategies, such as soft-templating synthesis using multiamines with an amphiphilic structure, ${ }^{16}$ surfactant-type organophosphates, ${ }^{17}$ polyhexamethylene biguanidine, ${ }^{18}$ hexadecylamine/ hexanol $^{19}$ or amphiphilic organosilanes, ${ }^{20}$ and hard-templating synthesis methods using carbon black ${ }^{20}$ or post-synthesis demetalation, ${ }^{21}$ have been reported to generate hierarchical SAPOs. Notably, Seo et al. ${ }^{16}$ have demonstrated that by designing bifunctional micropore- and mesopore-directing multiamine agents, it is possible to fabricate nanosheets of AFI, AEL, and ATO topologies with a thickness that can be altered by changing the number of amine groups. Kim et al. ${ }^{20}$ compared the catalytic performance of hierarchical SAPO-11 derived from hard-templating and organosilane templating routes, showing that isomer selectivity can only be enhanced by the presence of secondary mesopores and simultaneously suppressing the acidity at the external surface for samples prepared using the hard-templating route. On the one hand, hierarchical SAPO-11 compounds often outperform their conventional counterparts with enhanced isomer selectivity, as a result of reduced diffusion limitations. However, the known preparation methods are expensive and tedious, as they involve the preparation of homemade porogens, which requires rigorously controlled synthesis conditions. Moreover, poor control over crystal size and the pore-network structure, as well as a reduction of the crystallinity and acidity, or a disruption of the crystalline order are frequently encountered when introducing auxiliary porosity. For catalytic applications, ideal hierarchical zeolites should combine, in a single material, the inherent advantages of zeolitic materials, such as high crystallinity, strong acidity, and shape selectivity, with the advantageous mass-transfer properties of mesoporous materials. From an industrial viewpoint, it is desirable to design simple and scalable methods to fabricate hierarchical SAPOs with commercially available, low-cost starting materials. Besides, higher mass transfer demands a small primary particle size (smaller Thiele modulus) and an interconnected auxiliary macroporous/mesoporous system penetrating the crystal $^{22,23}$ or surrounding nanocrystals, ${ }^{24-26}$ as these are key structural factors affecting mass transport. ${ }^{27,28}$ Therefore, the objective for hierarchical SAPO synthesis is to build a zeotype architecture with small primary crystal size and well-connected auxiliary mesopores toward the external surface, while avoiding the use of expensive porogens and keeping the acid strength pertaining to their crystalline framework. This is particularly daunting for hierarchical SAPO synthesis; henceforth, only limited success has been accomplished to date, with respect to their zeolitic counterparts.

The present work provides a versatile orientated assembly strategy (OAS) to generate SAPO-11 with controlled hierarchical architecture from nanocrystalline building blocks. The synthesis is inspired by the insight that CPA dominates the dry gel conversion (DGC) for SAPO-11 synthesis, as will be briefly described in the first part of this work. Therefore, the DGC process can be interrupted deliberately to harvest sizefocused nanocrystallites of SAPO-11. In the second part of this work, we show that these nanocrystallites provide valuable platform building blocks, as they can be assembled as interlocking brick blocks to realize a wealth of hierarchical architectures. Intriguingly, these architectures are tunable with respect to coadditives, as the latter provide information about the crystallographic orientation of assembling nanocrystallites. A three-dimensional (3D) spongelike morphology and twodimensional (2D) assemblages of SAPO-11 nanosheets featuring a "house-of-cards" structure have been synthesized. Their structural, textural and acidic properties will be elucidated with suitable characterization techniques. In the last part of this paper, the catalytic performance of these hierarchical structures for the hydroisomerization of $n$-heptane will be assessed to discover the preferred architecture with the highest isomer selectivity. $n$-Heptane hydroisomerization is an exemplar of a bifunctional catalyzed process that manifests effects of diffusion over product distribution when the bifunctional catalysis is operated under ideal conditions, as proposed by Guisnet. ${ }^{29}$ A record-high isomer yield has been accomplished by one of the hierarchical SAPO-11 compounds. Kinetic and mass transfer measurements shall be employed to understand how auxiliary porosity influences isomer selectivity, and explained by postulating two regimes, corresponding to a different temperature range.

\section{EXPERIMENTAL SECTION}

Sample Preparation. The initial gel to synthesize SAPO11 was obtained as follows: $48.55 \mathrm{~g}$ of pseudoboehmite (PB, 75 wt $\% \mathrm{Al}_{2} \mathrm{O}_{3}$, Aluminum Corporation of China) was hydrolyzed in $257.57 \mathrm{~g}$ of deionized water while stirring. Then, $76.86 \mathrm{~g}$ of phosphoric acid ( $\geq 85.0$ wt \%, Shanghai Lingfeng Chemical Reagent Co., Ltd.) was added to the above mixture and homogenized overnight $(12 \mathrm{~h})$. Subsequently, $42.92 \mathrm{~g}$ of tetraethyl orthosilicate (TEOS, 28.0 wt $\% \mathrm{SiO}_{2}$, Shanghai Lingfeng Chemical Reagent Co., Ltd.) was introduced into the mixture, followed by the addition of $40.48 \mathrm{~g}$ of dipropylamine (DPA, >99 wt \%, TCI). The mixture was homogenized by stirring for an additional $5 \mathrm{~h}$, before 
transferring to a $353 \mathrm{~K}$ oil bath to evaporate the water solvent. The final molar composition of the synthesis dry gel was 1.2 DPA:1.0 $\mathrm{Al}_{2} \mathrm{O}_{3}: 1.0 \quad \mathrm{P}_{2} \mathrm{O}_{5}: 0.6 \mathrm{SiO}_{2}: 50 \mathrm{H}_{2} \mathrm{O}$. The obtained dry gel was grounded into a fine powder with a mortar and pestle before crystallization. A portion $(3.00 \mathrm{~g})$ of dry gel powder was introduced in a beaker placed in a Teflon-lined stainless steel autoclave; $10.00 \mathrm{~g}$ of water was poured outside the beaker, as a source of steam. The crystallization was conducted by steamassisted crystallization (SAC) at $473 \mathrm{~K}$ to undergo DGC. The process was monitored by quenching the crystallization process at varied time intervals and collecting intermediates for characterization.

To prepare prefabricated nanocrystallites, dry gel powder was precrystallized at $473 \mathrm{~K}$ for $2.5 \mathrm{~h}$ (labeled as SAPO-11-2.5h), collected and used as precursor for the synthesis of hierarchical SAPO-11 compounds. In a typical synthesis of hierarchical SAPO-11, $3.00 \mathrm{~g}$ of SAPO-11-2.5h, separated from the dry gel synthesis by washing and centrifugation, was mixed with an aqueous solution, prepared by mixing $2.25 \mathrm{~g}\left(0.45 \times 10^{-5} \mathrm{~mol}\right)$ of poly(diallyldimethylammonium chloride) (PDDA, $20 \mathrm{wt} \%$ aqueous solution, Aladdin) with $\mathrm{H}_{2} \mathrm{O}(50 \mathrm{~mL})$, homogenized under ambient conditions for $1 \mathrm{~h}$. The final composition of the mixture is $0.45 \times 10^{-5}$ PDDA:1.2 DPA:1.0 Al $\mathrm{Al}_{3}: 1.0 \quad \mathrm{P}_{2} \mathrm{O}_{5}: 0.6$ $\mathrm{SiO}_{2}: 50 \mathrm{H}_{2} \mathrm{O}$. The mixture was then transferred into a Teflonlined stainless steel autoclave and hydrothermally crystallized at $473 \mathrm{~K}$ for 2 days while tumbling $(50 \mathrm{rpm})$. The resultant product was washed with deionized water by centrifugation, dried at $373 \mathrm{~K}$ for $12 \mathrm{~h}$, and calcined at $823 \mathrm{~K}$ in a muffle oven for $10 \mathrm{~h}$. The product was denoted as SAPO-11-PDDA. All other hierarchical SAPO-11 compounds were synthesized in an analogous manner, except that one of the following chemicals was added as an additive to replace PDDA: $0.58 \mathrm{~g}\left(0.29 \times 10^{-2}\right.$ mol) of 1-ethyl-1-methylpyrrolidinium bromide (EMPB, 98 wt \%, Macklin) dissolved in $\mathrm{H}_{2} \mathrm{O}(20 \mathrm{~mL})$, a $20.0 \mathrm{~mL}$ ethanolic solution of $0.37 \mathrm{~g}\left(0.31 \times 10^{-2} \mathrm{~mol}\right)$ of tris(hydroxymethyl)methyl aminomethane (THAM, >99 wt \%, TCI), or a $20.0 \mathrm{~mL}$ ethanolic solution of $0.90 \mathrm{~g}\left(0.16 \times 10^{-2} \mathrm{~mol}\right)$ sucrose fatty acid esters (SFAE, 98 wt \%, Xiya Chemical Industry Co., Ltd., Shandong). In the latter two syntheses, to rule out the effect of ethanol, the mixture was first heated until it was dry, before replenishing with $\mathrm{H}_{2} \mathrm{O}$ to achieve the desired composition. Samples thus obtained were named SAPO-11-MEPB, SAPO11-THAM, and SAPO-11-SFAE, respectively, whereby the suffix represents the abbreviation for the additives. For comparison, another series of SAPO-11 compounds was synthesized using the same batches as those used for the hierarchical samples; however, the additives were added directly into the precursor solution to form a mixture before crystallization under hydrothermal synthesis conditions.

A conventional sample, for comparison, was synthesized under static conditions as frequently used. The molar composition of the synthesis gel was 1.2 DPA:1.0 $\mathrm{Al}_{2} \mathrm{O}_{3}: 1.0$ $\mathrm{P}_{2} \mathrm{O}_{5}: 0.6 \mathrm{SiO}_{2}: 50 \mathrm{H}_{2} \mathrm{O}$. A typical synthesis procedure was as follows: $1.46 \mathrm{~g}$ of pseudoboehmite and $2.31 \mathrm{~g}$ of phosphoric acid were hydrolyzed in deionized water under stirring for $12 \mathrm{~h}$. $1.29 \mathrm{~g}$ of TEOS was subsequently added to the mixture and stirred vigorously for $2 \mathrm{~h}$. Then, $1.21 \mathrm{~g}$ of DPA was slowly added and the mixture was stirred for $8 \mathrm{~h}$. The synthesis gel was hydrothermally crystallized at $453 \mathrm{~K}$ for 3 days under static conditions. The as-synthesized SAPO-11 was filtered, washed with deionized water, and dried at $393 \mathrm{~K}$ for $5 \mathrm{~h}$. Thereafter, the sample was calcined at $823 \mathrm{~K}$ for $15 \mathrm{~h}$. The obtained product was denoted as SAPO-11-C, with the suffix C standing for "conventional".

Pt replicas for pore-network determination were prepared following a previously reported procedure. ${ }^{27,28} \mathrm{~A}$ Pt replica was prepared by mixing $0.50 \mathrm{~g}$ of calcined SAPO- 11 with $0.30 \mathrm{~g}$ of $\mathrm{H}_{2} \mathrm{PtCl}_{6} \cdot 6 \mathrm{H}_{2} \mathrm{O}$ and $3.00 \mathrm{~g}$ water in a glass beaker, where it was stirred at $363 \mathrm{~K}$ for $8 \mathrm{~h}$. Subsequently, the solid was dried in a rotary evaporator and then heated at $673 \mathrm{~K}$ in $\mathrm{H}_{2}$ for $2 \mathrm{~h}$ to reduce the salt to metallic platinum. The molecular sieve framework was dissolved by aqueous hydrogen fluoride solution (HF), and the Pt replica was centrifuged, washed three times with water and ethanol each, and finally dried at $343 \mathrm{~K}$ for $6 \mathrm{~h}$. [Caution! HF is highly corrosive and proper protection measures should be taken!]

The Pt/SAPO-11 catalysts were prepared by incipient wetness impregnation of SAPO-11 with an aqueous solution of $\mathrm{H}_{2} \mathrm{PtCl}_{6}$, followed by drying and calcination at $673 \mathrm{~K}$ for $3 \mathrm{~h}$. The Pt content of each catalyst was kept at $0.5 \mathrm{wt} \%$ for all samples.

Characterization. The phase structure of the SAPO-11 was characterized by powder X-ray diffraction (XRD) patterns recorded on a Rigaku D/Max $2550 \mathrm{VB} / \mathrm{PC}$ diffractometer operating at $40 \mathrm{kV}$ and $100 \mathrm{~mA}$ with $\mathrm{Cu} \mathrm{K} \alpha(\lambda=1.5418 \AA)$ as an $\mathrm{X}$-ray source. The patterns were collected over a $2 \theta$ range from $3^{\circ}$ to $50^{\circ}$, with a scanning speed of $8^{\circ} \mathrm{min}^{-1}$. The size and morphological features of the samples were determined by means of scanning electron microscopy (SEM) images recorded with a NOVA Nano SEM450 microscope. Transmission electron microscope (TEM) micrographs were obtained using a JEM-2011 (JEOL) electron microscope setup. A drop of the examined solution was placed on a TEM grid covered by a perforated carbon film. Nitrogen adsorption and desorption isotherms were measured on an ASAP 2020 analyzer (Micromeritics, USA) at $77 \mathrm{~K}$, after samples were outgassed at $623 \mathrm{~K}$ under vacuum for $6 \mathrm{~h}$. The surface areas and pore volumes were determined using the Brunauer-Emmett-Teller (BET) method, and the Non-Local Density Functional Theory (NLDFT) method, respectively. Micropore volumes were derived from a $t$-plot approach, and the total pore volume values were estimated from the adsorbed quantity at a relative pressure $P / P_{0}=0.99$. Mercury intrusion tests were performed by using an AutoPore IV 9500 instrument. The intrusion volumes were measured at stepwise increasing pressures, equilibrated at each pressure step. The intrusion measurements started from a vacuum condition, and the extrusion measurements terminated at normal pressure. The pore size distribution was calculated according to the intrusion curves. A Micromeritics Autochem 2920 system was used for chemisorption measurements. The exposed active surface areas of the catalysts were determined via $\mathrm{H}_{2}$ titration at room temperature. In each experiment, $0.10 \mathrm{~g}$ of catalyst was used. The catalyst was reduced in $10 \% \mathrm{H}_{2} / \mathrm{Ar}$ with a flow rate of $30.0 \mathrm{~mL} \mathrm{~min}^{-1}$, ramped up at a rate of $5 \mathrm{~K} \mathrm{~min}^{-1}$ and then maintained at $673 \mathrm{~K}$ for $3 \mathrm{~h}$, before catalytic assessment. After reduction, the catalyst was purged with an ultrahigh-purity helium flow, before being cooled to room temperature. $\mathrm{H}_{2}$ pulses were introduced over the reduced catalyst and the $\mathrm{H}_{2}$ uptake for each pulse was monitored using a thermal conductivity detector (TCD). Solid-state NMR measurements were performed using an Agilent DD2-500 MHz spectrometer operating at a magnetic field strength of $11.7 \mathrm{~T} .{ }^{27} \mathrm{Al}$ MAS NMR spectra were recorded at $130.2 \mathrm{MHz}$ with a spinning rate of 13 $\mathrm{kHz}, 200$ scans, and $2 \mathrm{~s}$ recycle delay. The chemical shifts were 

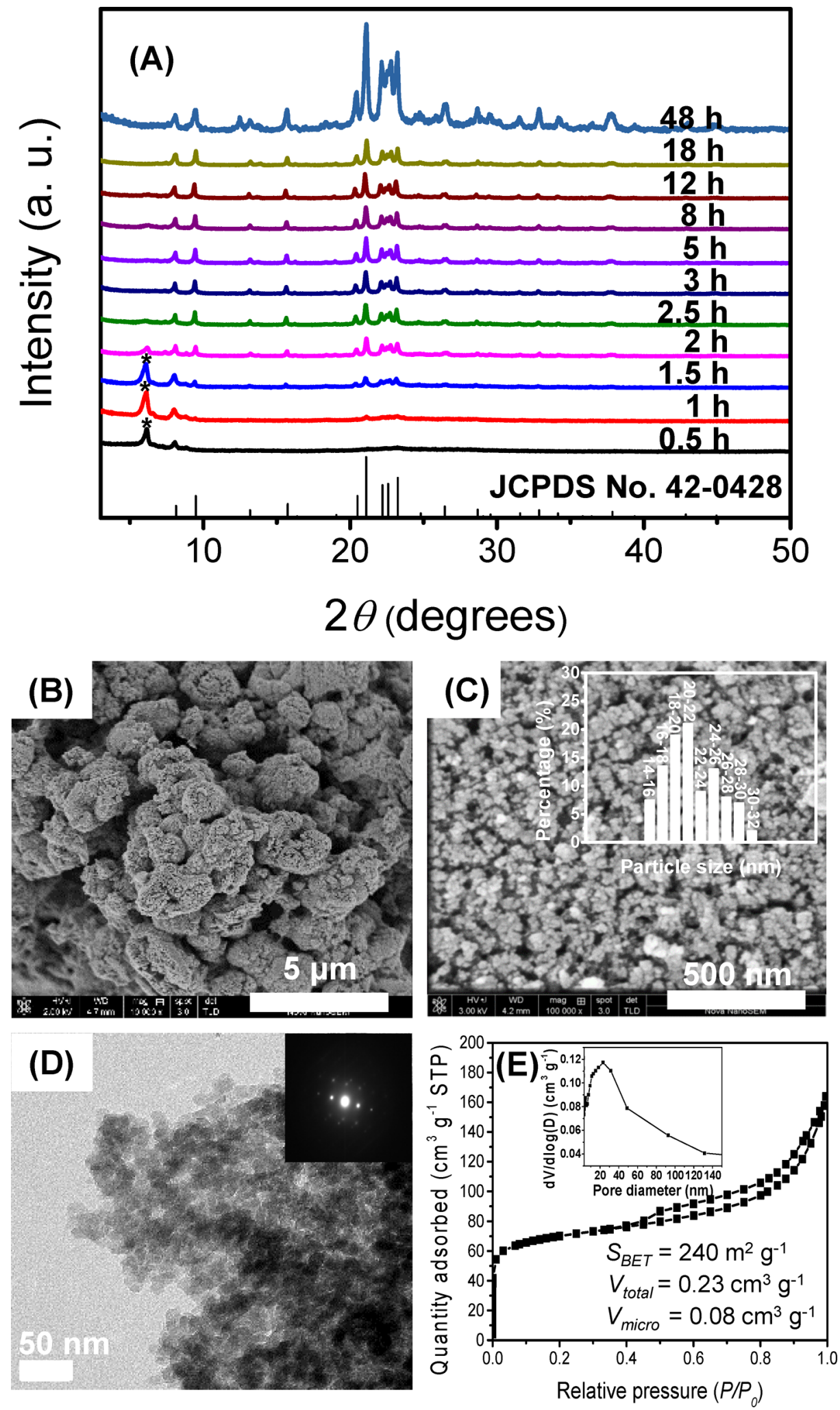

Figure 1. (A) Time-dependent X-ray diffraction (XRD) patterns of SAPO-11 in steam-assisted crystallization (SAC), which is a type of DGC synthesis. (B, C) Scanning electron microscopy (SEM) and (D) transmission electron microscopy (TEM) micrographs for as-synthesized SAPO11-2.5h. (E) $\mathrm{N}_{2}$ physisorption isotherm for calcined SAPO-11-2.5h (inset of (E) shows the mesopore size distribution; the asterisk (*) in (A) indicates the DPA $\cdot \mathrm{H}_{3} \mathrm{PO}_{4}$ phase).

referenced to $1.0 \mathrm{wt} \% \mathrm{Al}\left(\mathrm{NO}_{3}\right)_{3}$ aqueous solution. ${ }^{31} \mathrm{P}$ MAS NMR experiments with high power proton decoupling were conducted at $202.3 \mathrm{MHz}$ with a spinning rate of $14 \mathrm{kHz}, 20$ scans, and a 10-60 s recycle delay. The chemical shifts were referenced to 85 wt $\% \mathrm{H}_{3} \mathrm{PO}_{4} \cdot{ }^{29} \mathrm{Si}$ MAS NMR spectra with high power proton decoupling were recorded at $99.3 \mathrm{MHz}$ with a spinning rate of $4 \mathrm{kHz}, 600$ scans, and a recycle delay of $10-$ 300 s. The elementary composition for powder products and 
Scheme 1. (a) Proposed Formation of SAPO-11 in DGC Synthesis through Orientated Attachment of Particulates Intermediates, and the Synthesis Strategies for the Orientated Assembly of Prefabricated Nanocrystallites with Growth Modifiers (b) PDDA, (c) MEPB, (d) THAM, and (e) SFAE, To Construct Diverse Architectures

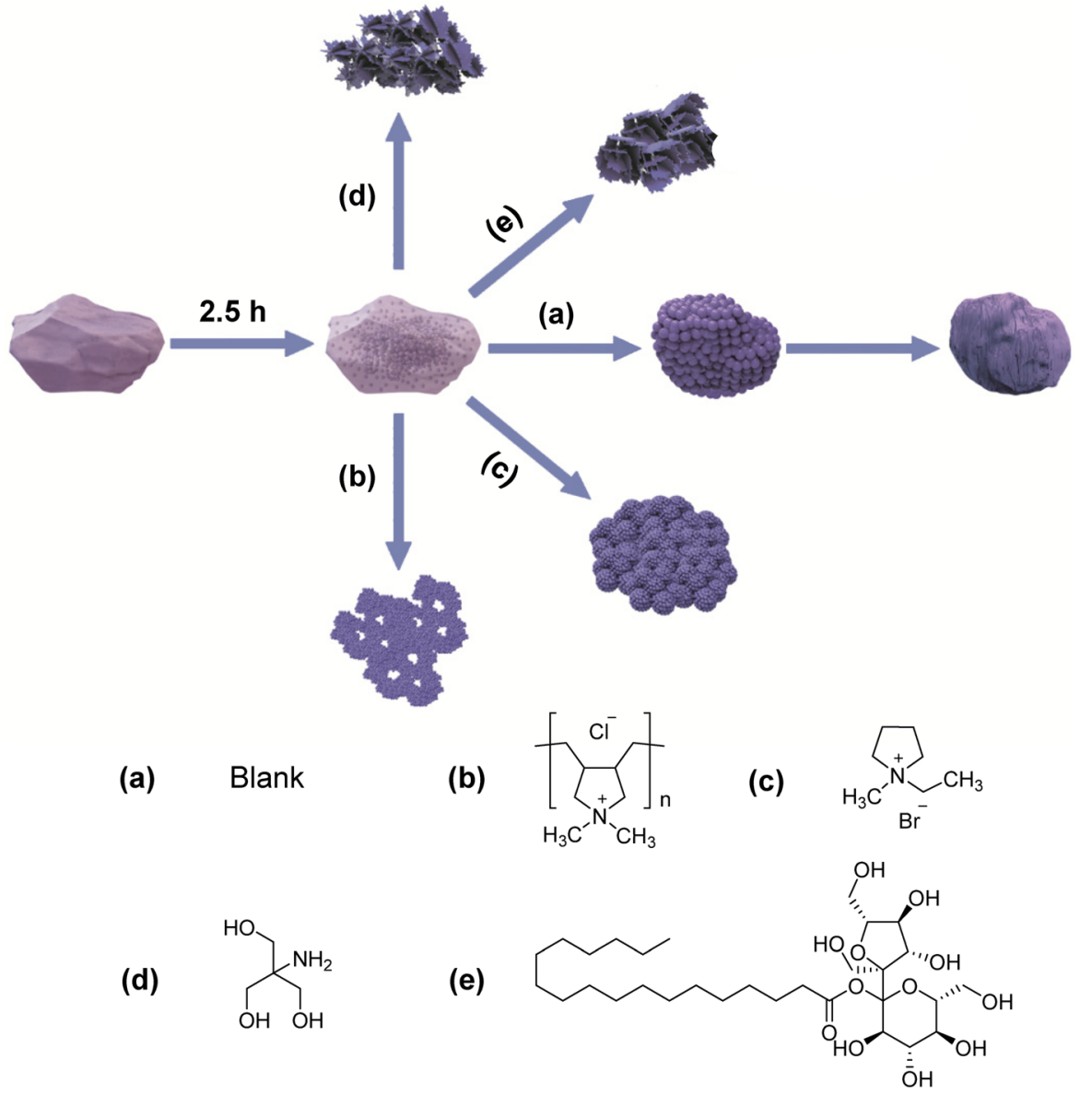

mother liquor were measured by inductively coupled plasmaoptical emission spectrometry (ICP) on an IRIS 1000 instrument. The acidity of SAPO-11 was probed by alkaline molecules (pyridine (Py) or 2,4,6-collidine (Coll)) desorption infrared (Py-IR, Coll-IR) spectra on a Spectrum 100 FT-IR spectrometer (Nicolet Co., USA). All samples were pressed into self-supporting wafers $($ diameter $=1.4 \mathrm{~cm}$, weight $=166$ $\mathrm{mg})$ and were preheated at $723 \mathrm{~K}$ for $4 \mathrm{~h}$ under vacuum $(1.3 \times$ $\left.10^{-2} \mathrm{~Pa}\right)$. The background baselines of the samples were collected and subtracted at room temperature. The amount of adsorbed probe molecules was calculated from the integrated area of the given bands with their distinct molar extinction coefficients $\left(\varepsilon_{\mathrm{H} \text {-bond }}\left(1638 \mathrm{~cm}^{-1}\right)=1.9 \mathrm{~cm}^{-1} \mathrm{~mol}^{-1}, 30\right.$ $\varepsilon_{\text {Brønsted }}\left(1545 \mathrm{~cm}^{-1}\right)=1.67 \mathrm{~cm} \mu \mathrm{mol}^{-1}, \varepsilon_{\text {Lewis }}\left(1455 \mathrm{~cm}^{-1}\right)=$ $\left.2.22 \mathrm{~cm} \mu \mathrm{mol}^{-1}\right){ }^{31}$ The diffusivity of 2 -methylhexane in the SAPO-11 samples was measured by gravimetric analysis, using an intelligent gravimetric analyzer instrument (Model IGA-100, Hiden Analytical, Ltd., Warrington, U.K.). The sensitivity of the balance is $1 \mu \mathrm{g}$. The buoyancy correction following from Archimedes' principle is considered to yield the true weight. The calcined sample was pretreated at $573 \mathrm{~K}$ in a $\mathrm{N}_{2}$ atmosphere for $3 \mathrm{~h}$ to remove adsorbed impurities, and the absence of impurities during this procedure was indicated by the constant weight of the sample. Afterward, the sample was cooled to $365 \mathrm{~K}$. The diffusivity measurement was initiated by replacing the $\mathrm{N}_{2}$ stream with a known amount of gaseous mixture, consisting of 2-methylhexane and $\mathrm{N}_{2}$, controlled through a precalibrated mass flowmeter (by flowing $\mathrm{N}_{2}$ saturated with 2 -methylhexane at $288 \mathrm{~K}$ ). The feed was maintained until the sample was equilibrated, as indicated by reaching a constant mass.

Catalytic Activity Tests. The hydroisomerization of $n$ heptane was carried out in a fixed-bed flow reactor at atmospheric pressure. The catalyst loading was $1.2 \mathrm{~g}$ (40-60 mesh). Prior to reaction, the catalyst was reduced in $\mathrm{H}_{2}$ flow $\left(23 \mathrm{~mL} \mathrm{~min}^{-1}\right)$ at $673 \mathrm{~K}$ for $3 \mathrm{~h}$, then cooled to the reaction temperature under $\mathrm{H}_{2}$ flow. $n$-Heptane was introduced to the reactor using a constant-flux pump, which gave a $\mathrm{H}_{2} / n$-heptane molar ratio of 5 . The weight hourly space velocity (WHSV) of $n$-heptane was $1.0 \mathrm{~g}_{n \text {-heptane }}\left(\mathrm{g}_{\text {cat }} \mathrm{h}\right)^{-1}$. The product gas mixture was analyzed periodically with an online gas chromatograph that was equipped with a flame ionization detection (FID) device and a HP-PONA capillary column $(50 \mathrm{~m} \times 0.2 \mathrm{~mm} \times$ $0.5 \mu \mathrm{m})$. The oven temperature was maintained at $343 \mathrm{~K}$.

Kinetic Experiments. The activation energies for catalytic hydroisomerization of $n$-heptane were measured in the same fixed-bed reactor. A catalyst with a mass of $0.025-0.15 \mathrm{~g}$ (40$60 \mathrm{mesh}$ ) was diluted with the same mass of quartz sand. The $n$-heptane was introduced into the reactor by a constant-flux pump. The gas flow was between $23 \mathrm{~mL} \mathrm{~min}^{-1}$ and $92 \mathrm{~mL}$ $\min ^{-1}$. The $n$-heptane consumption rates were measured at temperatures between $473 \mathrm{~K}$ and $673 \mathrm{~K}$, and the conversion was kept below $10 \%$ for all measurements. The platinum 
loading was 0.5 wt \% for all kinetic experiments, to guarantee that the rate of reaction is independent of platinum loading. ${ }^{10,29}$

\section{RESULTS AND DISCUSSION}

Studies of the crystallization process showed that the nonclassical CPA predominates in the zeolite crystallization process under certain circumstances. ${ }^{4,5,8,32}$ In particular, Bein et al. $^{4}$ and our group ${ }^{5}$ found that, under high supersaturation, synthesis systems, such as DGC for zeolite beta, the lack of liquid water minimizes the contribution from classic atom-byatom growth and CPA becomes the main crystallization route. Under high supersaturation or concentrated media, the size of the nuclei and their distribution can be conveniently controlled, as a result of a high nucleation rate and short nucleation time span. ${ }^{33,34}$ In the current investigation, we have monitored the structural evolution of SAPO-11 in DGC by using X-ray diffraction (XRD) (Figure 1A) and scanning electron microscopy (SEM) (see Figure $\mathrm{S} 1$ in the Supporting Information). The time-dependent XRD patterns show that the initial dry gel possesses weak diffraction peaks can be assigned to the formed DPA $\cdot \mathrm{H}_{3} \mathrm{PO}_{4}$ phase, which may interact with the $\mathrm{Al}$ species, ${ }^{35}$ so that the gel progressively transforms to SAPO-11 (JCPDS File No. 42-0428). SEM micrographs demonstrate that an irregularly shaped dry gel undergoes decomposition into amorphous particulates, the formation of tiny aggregates of nanocrystallites, and their subsequent fusion through CPA into a bulk crystal. The SEM images of the final product after $72 \mathrm{~h}$ crystallization show well-defined crystal shapes; grain boundaries have vanished, as a result of attached growth (Figure S1L). The lack of liquid phase water may have inhibited the growth of SAPO-11 through the classical atom-byatom growth at the crystal/liquid interface. Instead, high concentration and nucleation rates favor bursts of nucleates. Consequently, nanocrystallites attaching and fusing with neighboring ones to form a bulk crystal becomes a dominant crystallization route, as dissolution of nanocrystallites and their growth through dissolution-crystallization is disfavored in the absence of liquid phase water, which has also been observed in other dry gel syntheses. ${ }^{4,5,36,37}$ All of this is evidence for CPA as the predominant route in the DGC of SAPO-11. The growth process is graphically depicted in Scheme 1a.

From the above experiment, it has been convincingly shown that, for the $2.5 \mathrm{~h}$ steam-treated sample in DGC (named SAPO-11-2.5h), the precursor phase has been completely consumed and some characteristic diffraction peaks corresponding to an AEL topology become discernible. The corresponding SEM micrographs (Figures $1 \mathrm{~B}$ and $1 \mathrm{C}$ ) exhibit size-focused particulates between $15 \mathrm{~nm}$ to $30 \mathrm{~nm}$, albeit that they are ill-defined in shape. Both the XRD patterns (Figure 1A) and TEM micrograph (Figure 1D), together with the selected-area electron diffraction (SAED) pattern (inset of Figure 1D) consistently show that these particulates are crystalline and are loosely packed to form aggregates. Part of the sample was calcined to measure $\mathrm{N}_{2}$ physisorption isotherms, in order to determine the textural properties, which are displayed in Figure 1E. In agreement with the SEM observations, the isotherms for calcined SAPO-11-2.5h clearly exhibit a combination of type I and type IV isotherms, with a type $\mathrm{HI}$ hysteresis loop. The filling by $\mathrm{N}_{2}$ appearing at $P$ / $P_{0}<0.1$ evidence the presence of micropores, and the jump in uptake of $\mathrm{N}_{2}$ at $P / P_{0}>0.8$ is ascribed to capillary condensation, because of the coexistence of mesopores. The sample thereby contains both micropores and mesopores, and a mesopore size distribution (inset of Figure 1E) deduced from the adsorption branch of the physisorption isotherm implies a rather broad pore size distribution, between $4 \mathrm{~nm}$ and $100 \mathrm{~nm}$, centered at ca. $25 \mathrm{~nm}$. The micropores originate from the crystalline framework of AEL topology, and the mesopores can be attributed to the voids within the particulate aggregates.

The above observation inspires us to use SAPO-11-2.5h as building blocks to construct hierarchical structures. The SAPO$11-2.5 \mathrm{~h}$ was selected because DPA. $\mathrm{H}_{3} \mathrm{PO}_{4}$ phase had been completely consumed at this stage of crystallization, and evenly distributed nanocrystallites of AEL topology had formed. It is speculated that these nanocrystallines building blocks can grow spontaneously in the presence of occluded DPA without circumventing the hurdles for nucleation. In addition, the loose packing characteristic will facilitate the manipulation of them as building blocks, since there are no energetic hurdles for them to grow or assemble, and the crystalline nature suggests that these particulates have low solubility in weak basic or neutral synthesis media.

Synthesis and Structural Characterizations of Hierarchical SAPO-11 Compounds. To systematically investigate the growth of distinct hierarchical architectures, we assembled the premanufactured SAPO-11-2.5h in the presence of four typical growth modifiers, namely, polymeric PDDA, molecular MEPB, and surfactants such as THAM and SFAE. After homogenization through stirring under ambient conditions, each mixture was introduced into a Teflon-lined stainless steel autoclave to undergo complete crystallization. It is noteworthy that no more DPA was added before resuming crystallization, so that the medium has a close to neutral $\mathrm{pH}$ level (with a measured value of ca. 7) that would not cause substantial dissolution of premanufactured nanocrystallites. The crystallization was conducted under agitation for $48 \mathrm{~h}$. Because the mean particle size for SAPO-11-2.5h exceeds $15 \mathrm{~nm}$, which is beyond the size of inorganic particles that are suitable for selfassembly (due to the relatively small interfacial area and large weight), we have adopted a tumbling crystallization synthesis process to promote the contact of additives with these particulates. Precipitation due to the large particle size can be minimized, and interaction between loosely packed particulates is weakened by tumbling. Tumbling also facilitates mixing of the ingredients and ensures homogeneity of the products. The fully crystallized products derived from diverse growth modifiers are denoted as SAPO-11-X, whereby the suffix denotes the additives. The obtained powders were calcined and extensively characterized using a combination of techniques.

As discussed later, different morphologies are obtained, which are dependent on the modifier, as schematically illustrated in Scheme 1. For SAPO-11-PDDA, Figure 2 displays the XRD pattern, together with a conventional sample juxtaposed for comparison (Icm2, JCPDS File No. 42-0428; $a$ $=13.35 \AA, b=18.66 \AA, c=8.44 \AA) .^{38}$ The diffraction lines coincide with the AEL topology, and all samples are free from impurity phases, showing that the addition of growth inhibitors has a negligible effect on their crystallinity or purity. In addition, the diffraction peaks are broadened in the hierarchical samples, suggesting a reduced crystal size, as a result of inhibited growth by the presence of additives.

A panoramic SEM image of SAPO-11-PDDA (Figure 3A) confirms that this material is comprised of large, spongelike crystals $2-3 \mu \mathrm{m}$ in size. A larger magnification (Figure $3 \mathrm{~B}$ ) displays tiny aggregates of nanocrystals, ranging from $20 \mathrm{~nm}$ to $30 \mathrm{~nm}$, which also agrees well with the TEM observations 


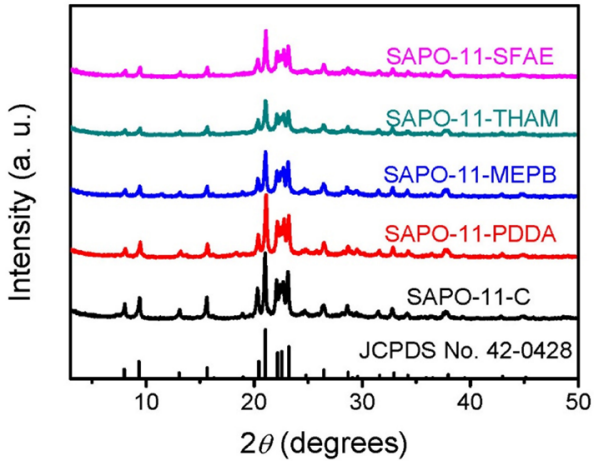

Figure 2. XRD patterns of hierarchical SAPO-11 compounds derived from various organic additives as the growth modifier, together with a conventional SAPO-11-C for comparison.

(Figure $3 \mathrm{C}$ ). It is noteworthy that the primary particle size is close to that of SAPO-11-2.5h, suggesting that PDDA inhibits SAPO-11 crystallites against growth by particle attachment or Ostwald ripening. PDDA is a typical cationic polyelectrolyte that interacts with negatively charged inorganic particles through Coulombic attraction, which is often stronger than the interparticle interaction. When PDDA was loaded into the precursor mixture for hydrothermal synthesis, PDDA might have enclosed the inorganic precursors in the same way and become an obstacle for their dissolution and crystallization. Noteworthy, for SSZ-13 crystallization, ${ }^{8}$ which is an aluminosilicate (zeolite) that crystallizes in more-basic media, the role of PDDA may be similar, but the influence on product morphology is different. PDDA may encapsulate the inorganic precursors and restrict their transformation to wormlike particles; consequently, a slow time release of inorganic species into soluble aqueous solution is still possible, but it becomes too slow for the relatively high $\mathrm{OH}^{-}$concentration, in comparison with SAPO-11 synthesis media, which eventually promotes atom-by-atom classical growth and, instead, leads to the formation of bulky crystals. SAED (see the inset of Figure $3 \mathrm{C}$ ) shows a spotlike pattern, corresponding to the features of a single-crystalline material, lending further evidence for its crystalline nature and the alignment of neighboring particulates. The connectivity of the pore network is demonstrated by the TEM micrograph of the Pt replica of the same sample, prepared by nanocasting and removal of the SAPO framework by HF dissolution. ${ }^{27,28}$ Metallic platinum is stable under the electron beam and can honestly reflect the mesopore/macropore architecture of the parent material from which it is casted. The $\mathrm{Pt}$ replica of SAPO-11-PDDA in Figure 3D shows interconnected $\mathrm{Pt}$ nanoparticles between $15 \mathrm{~nm}$ and $25 \mathrm{~nm}$, which proves that the mesopores of the parent material are interconnected. ${ }^{39}$ As observed for the SAPO-11-2.5h sample, the isotherm combines the features of type I and type IV isotherms (Figure 4A). Again, a steep uptake of $\mathrm{N}_{2}$ occurs at $P$ / $P_{0}<0.1$, resulting from the filling of the micropores. In addition, a follow-up jump in uptake at $P / P_{0}=0.8$ due to capillary condensation is observed, confirming the presence of both micropores and mesopores. The corresponding pore size distribution, derived from the adsorption branch of the isotherm, is shown in Figure $4 \mathrm{~B}$, exhibiting pores ranging in diameter between $5 \mathrm{~nm}$ and $125 \mathrm{~nm}$, with a median size at ca. $30 \mathrm{~nm}$. The median mesopore size $(30 \mathrm{~nm}$ vs $25 \mathrm{~nm})$ and total pore volume $\left(0.23 \mathrm{~cm}^{3} \mathrm{~g}^{-1}\right.$ vs $\left.0.37 \mathrm{~cm}^{3} \mathrm{~g}^{-1}\right)$ are larger than
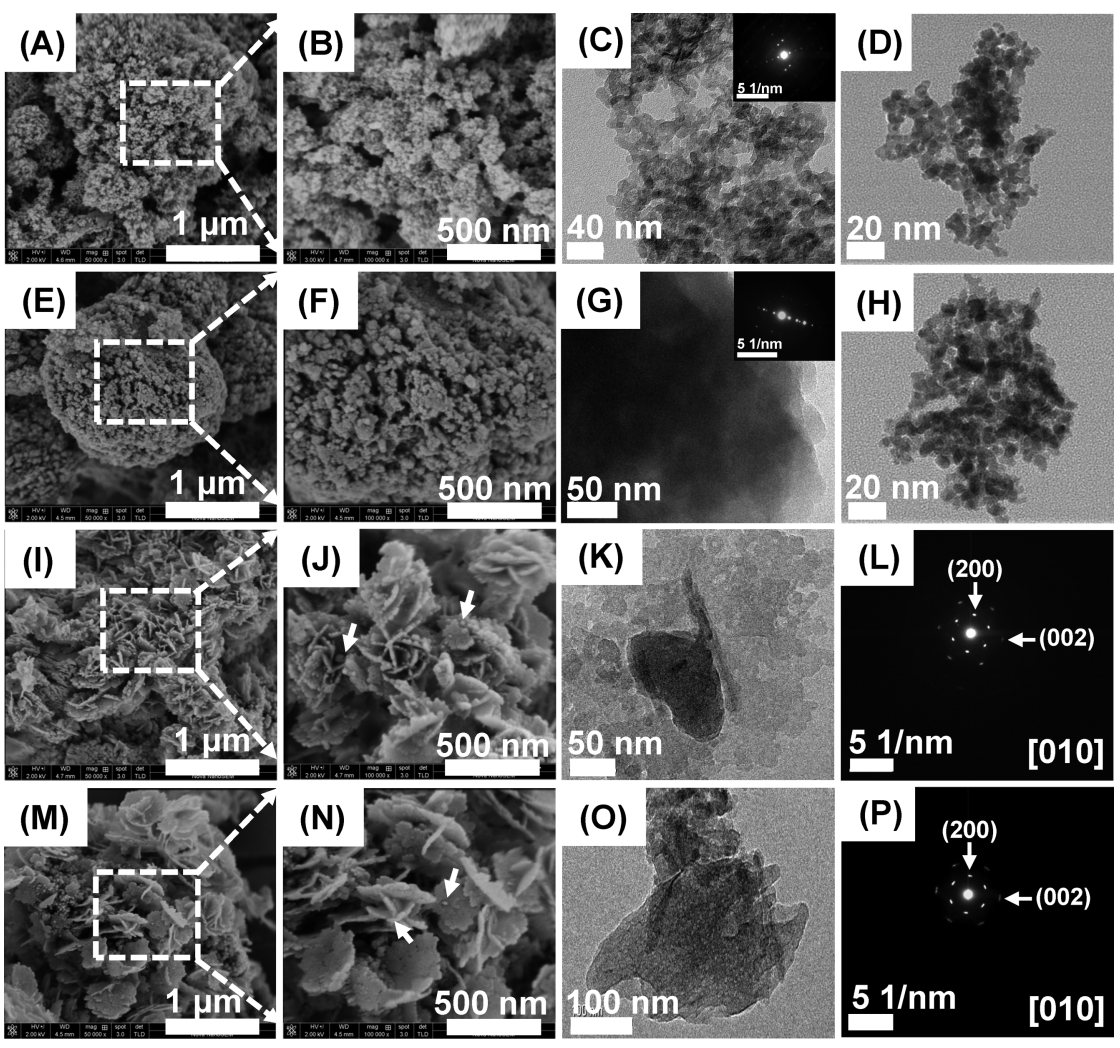

Figure 3. (A, B) SEM, (C) TEM, and (D) Pt replica images of SAPO-11-PDDA; (E, F) SEM, (G) TEM, and (H) Pt replica images of SAPO-11MEPB; (I, J) SEM, (K) TEM images, and (L) corresponding SAED pattern of SAPO-11-THAM; (M, N) SEM, (O) TEM images, and (P) associated SAED pattern of SAPO-11-SFAE. 

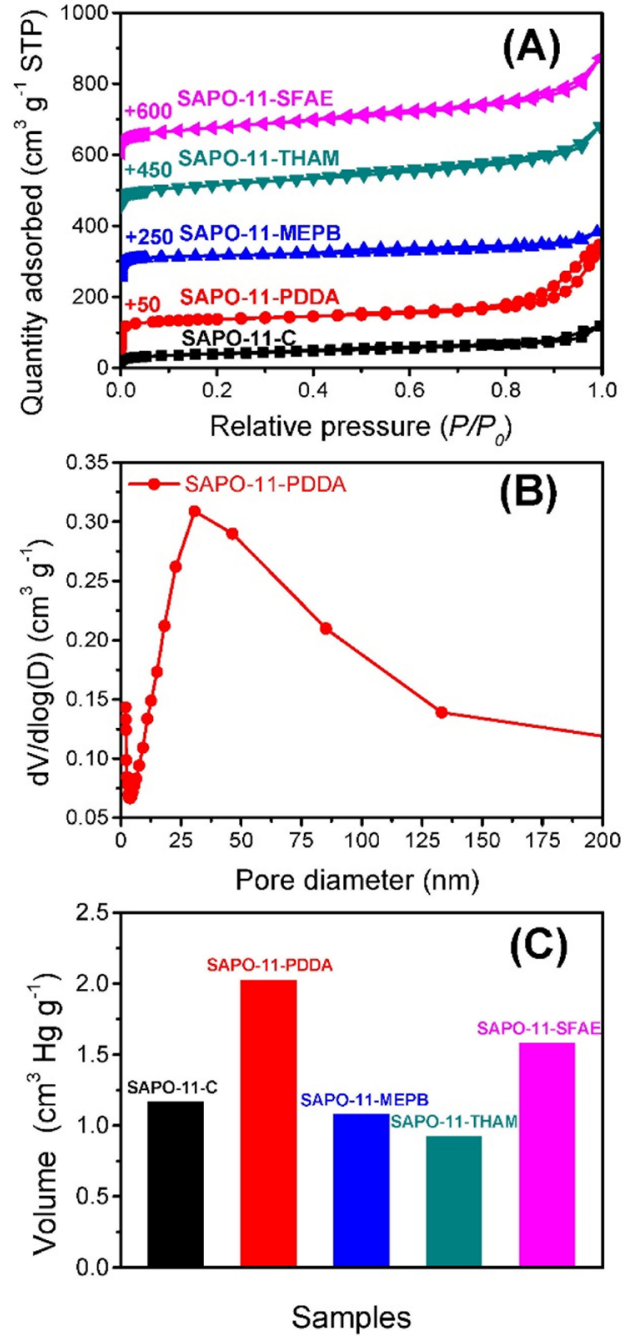

Figure 4. (A) $\mathrm{N}_{2}$ adsorption and desorption isotherms for SAPO-11 compounds, (B) the pore size distribution for SAPO-11-PDDA corresponding to the $\mathrm{N}_{2}$ adsorption isotherm, and (C) pore volumes obtained from mercury intrusion measurements for SAPO-11 compounds.

those of SAPO-11-2.5h, showing that PDDA has enlarged the mesopores and macropores between the nanocrystallite building blocks during the assembly and crystallization process. Some of these auxiliary pores exceed the mesopore region of 50 $\mathrm{nm}$ and should be regarded as macropores. However, contrary to SAPO-11-2.5h, the absence of a forced closure at $P / P_{0}=$ 0.45 for the desorption branch of the isotherm indicates that the mesopores are directly connected to each other without narrow constrictions between them..$^{22,23,40}$ In comparison with known hierarchical SAPO-11 compounds, the primary particles are smaller and more uniform, and the mesopores are more open toward the external surface. ${ }^{17,20}$ SAPO-11-PDDA has the largest BET surface area $\left(302 \mathrm{~m}^{2} \mathrm{~g}^{-1}\right)$ and pore volume among the synthesized SAPO-11-X materials (Table 1). A complementary mercury intrusion porosimetric measurement (see Figure 4C and Figure S2C in the Supporting Information) demonstrates that there are both mesopores and very large macropores $\left(>10^{3} \mathrm{~nm}\right)$ in the sample. A total macropore and mesopore volume of ca. $2.0 \mathrm{~cm}^{3} \mathrm{~g}^{-1}$ is measured (in sharp contrast to conventional SAPO-11-C, Figure 4C and Figure $\mathrm{S} 2 \mathrm{~B}$ in the Supporting Information), suggesting a large number of pores above $4 \mathrm{~nm}$ that are accessible by mercury through the external surface. ${ }^{2,41}$ The advantages of small primary particulates and enhanced pore connectivity are attributed to the inhibited growth of SAPO-11 crystals in the presence of PDDA, which covers their surface, and the cross-linking polymeric backbone, which facilitates formation of penetrating macropores and mesopores, respectively. Compared with other hierarchical material architectures, a high level of control over crystal size and pore structure has been accomplished through this novel synthesis route. ${ }^{2,42,43}$ In short, SAPO-11-PDDA has a highly crystalline hierarchical structure composed of tiny particulates, and is rich in interconnected macroporosity and mesoporosity that extends throughout the crystal and is open toward the external surface.

SAPO-11-MEPB has a distinct assembled morphology, as disclosed by SEM micrographs (Figures $3 \mathrm{E}$ and $3 \mathrm{~F}$ ). It consists of evenly distributed agglomerates of $2-4 \mu \mathrm{m}$ with corrugated surfaces. SEM micrographs show that the roughness is mainly caused by the agglomeration of primary building blocks of 20$30 \mathrm{~nm}$, some of which reach $100 \mathrm{~nm}$, as a result of growth by attachment of primary building blocks. However, the typical TEM micrograph, together with its corresponding SAED pattern, advocates that neighboring primary building blocks are mutually aligned (Figure $3 G$ ). In some parts, the fusion of primary particulates has caused their tight agglomeration into bulky ones $(\sim 100 \mathrm{~nm})$. A TEM micrograph of a Pt replica of SAPO-11-MEPB suggests that the voids are partly interconnected (Figure $3 \mathrm{H}$ ), and the size of the Pt particles is within the scale range of mesopores. $\mathrm{N}_{2}$ physisorption (Figure $4 \mathrm{~A}$ ) of SAPO-11-MEPB shows a type I isotherm at $P / P_{0}<0.1$, so that the sample mainly contains micropores. The absence of $\mathrm{N}_{2}$ uptake due to capillary condensation suggests the absence of mesopores. Complementary mercury porosimetry of SAPO-11MEPB corroborates that mesoporosity in this sample is insignificant, which is similar to a conventional sample (Figure 4C and Figures S2A and S2D in the Supporting Information).

Table 1. Textural Properties of Conventional and Hierarchical SAPO-11 Compounds Derived from $\mathrm{N}_{2}$ Physisorption Isotherms, Platinum Dispersion on the Supported Catalysts, and Molar Composition of the Samples

\begin{tabular}{|c|c|c|c|c|c|c|c|}
\hline sample & $S_{\mathrm{BET}}\left(\mathrm{m}^{2} \mathrm{~g}^{-1}\right)^{a}$ & $S_{\text {micro }}\left(\mathrm{m}^{2} \mathrm{~g}^{-1}\right)^{b}$ & $S_{\text {ext }}\left(\mathrm{m}^{2} \mathrm{~g}^{-1}\right)^{c}$ & $V_{\text {total }}\left(\mathrm{cm}^{3} \mathrm{~g}^{-1}\right)$ & $V_{\text {micro }}\left(\mathrm{cm}^{3} \mathrm{~g}^{-1}\right)^{c}$ & Pt dispersion ${ }^{d}\left(\mathrm{Pt} / \mathrm{H}^{+}, \%\right)$ & molar composition ${ }^{e}$ \\
\hline SAPO-11-C & 134 & 101 & 33 & 0.18 & 0.05 & $48.7(0.055)$ & $\mathrm{Al}_{0.85} \mathrm{Si}_{0.11} \mathrm{P}_{0.68} \mathrm{O}_{0.90}$ \\
\hline SAPO-11-PDDA & 302 & 157 & 145 & 0.37 & 0.11 & $73.0(0.077)$ & $\mathrm{Al}_{0.70} \mathrm{Si}_{0.08} \mathrm{P}_{0.58} \mathrm{O}_{0.74}$ \\
\hline SAPO-11-MEPB & 221 & 126 & 95 & 0.21 & 0.07 & $54.9(0.095)$ & $\mathrm{Al}_{0.70} \mathrm{Si}_{0.11} \mathrm{P}_{0.58} \mathrm{O}_{0.75}$ \\
\hline SAPO-11-THAM & 226 & 115 & 111 & 0.36 & 0.06 & $58.2(0.045)$ & $\mathrm{Al}_{0.85} \mathrm{Si}_{0.09} \mathrm{P}_{0.68} \mathrm{O}_{0.89}$ \\
\hline SAPO-11-SFAE & 266 & 117 & 149 & 0.42 & 0.09 & $60.2(0.049)$ & $\mathrm{Al}_{0.70} \mathrm{Si}_{0.13} \mathrm{P}_{0.55} \mathrm{O}_{0.75}$ \\
\hline
\end{tabular}

${ }^{a}$ Calculated by the BET method in the $P / P_{0}$ range of $0.05-0.25 .{ }^{b}$ Deduced using the $t$-plot method. ${ }^{c}$ Inferred from $S_{\text {ext }}=S_{\mathrm{BET}}-S_{\text {micro. }}{ }^{d} \mathrm{Pt}$ dispersion determined by chemical titration of $\mathrm{H}_{2}$ after supporting $0.5 \mathrm{wt} \% \mathrm{Pt}, \mathrm{Pt} / \mathrm{H}^{+}$is the ratio of the number of accessible platinum atoms to the number of Brønsted acid sites. ${ }^{e}$ Molar composition determined by inductively coupled plasma (ICP) analysis. 
MEPB has led to an assembly of nanocrystallites, only the size of which has been partly maintained. Unlike PDDA, which has more positively charged binding sites to bind to the nanocrystallite surface, MEPB only has one binding site and could be expelled from the nanocrystallite surface when neighboring nanocrystallites aggregate and fuse into a bigger crystal. As fusion reduces the interfacial energy by eliminating grain boundaries, bulky crystals can form inside the sample. Coagulation due to the adsorption of positively charged MEPB on the negatively charged nanocrystallite surface (as a consequence of $\mathrm{Si}$ substitution by $\mathrm{P}$ ) might accelerate this process. In short, MEPB does not bring about mesopores, but only roughens the external surface of the assembled architecture, with a concurrent increase in BET surface area, up to $221 \mathrm{~m}^{2} \mathrm{~g}^{-1}$ (Table 1), while fusion of the building blocks inside the assembled structure is also detected.

For SAPO-11-THAM, generated from a smaller molecular inhibitor THAM, which possesses three hydroxyl end groups and one amine group (see Scheme 1), it is intriguing to find that the primary particulates have unidimensionally organized into a "house-of-cards" structure of nanosheets (Figures 3I-L), somewhat reminiscent of a gypsum desert rose. A panoramic image for SAPO-11-THAM discloses that the nanosheets are uniform in thickness (ca. $20 \mathrm{~nm}$ ), with an average lateral size of $2-5 \mu \mathrm{m}$. From an SEM image of the edge-on nanosheet, it is estimated that the average thickness of the sheets is identical to the particle size of SAPO-11-2.5h. At the verges of the nanosheets, primary building blocks (indicated by arrows in Figures $3 \mathrm{~J}$ and $3 \mathrm{~N}$ ), ranging from $20 \mathrm{~nm}$ to $30 \mathrm{~nm}$ in size, can be found, suggesting a mosaic-like orientated assembly history for their formation. The sample has been sonicated to obtain a detached piece to determine the surface crystallographic orientation in a direction normal to the sheets. An SAED pattern (Figure 3L) has been measured for one sheet laying on the copper grid, showing that the $b$-axis is perpendicular to the sheets (diffraction peaks for $h+k+l=$ odd are systematically absent for the body-centered Icm 2 structure). In the AEL structure, the most commonly obtained major zone axis orientation runs along the $b$-axis. ${ }^{38}$ SAPO-11-THAM $\left(226 \mathrm{~m}^{2}\right.$ $\mathrm{g}^{-1}$ ) possesses a large surface area, with respect to the conventional SAPO-11-C $\left(134 \mathrm{~m}^{2} \mathrm{~g}^{-1}\right)$, but no appreciable mesoporosity is found, as shown in Table 1, Figures $4 \mathrm{~A}$ and $4 \mathrm{C}$, and Figure S2E in the Supporting Information.

SAPO-11-SFAE, synthesized using SFAE as the additive, has a morphology close to that of SAPO-11-THAM. The SEM/ TEM images and SAED pattern for a piece of SAPO-11-SFAE sheet corroborate that the structures are almost identical to each other (Figure 3M-P). However, the SAPO-11-SFAE has a slightly higher surface area $\left(266 \mathrm{~m}^{2} \mathrm{~g}^{-1}\right.$ vs $226 \mathrm{~m}^{2} \mathrm{~g}^{-1}$ ) (see Table 1 and Figure 4A) and the structure is more uniform. As the sample is fragile under the electron beam during TEM measurements, it is difficult to collect a high-resolution transmission electron microscopy (HRTEM) micrograph. A representative one taken at short beam contact time is shown in Figure S3 in the Supporting Information. The corresponding HRTEM micrograph clearly shows the lattice fringe associated with the $b$-axis of the AEL topology. The stacking of individual sheets can be inhibited by this additive, which is also a surfactant, and a large surface area is retained. It is interesting that a "houses-of-cards" structure composed of nanosheets is produced for the two additives interacting with nanocrystallites, not through strong Coulomb attraction, but through weak adsorption. It seems that the nanocrystallites tend to form sheetlike, aligned superstructures when they approach each other, while the presence of additives hinders the stacking of the nanosheets. The $\mathrm{N}_{2}$ physisorption isotherms and mercury porosimetric measurements show the near-absence of mesopores and predominance of macropores (see Figures $4 \mathrm{~A}$ and $4 \mathrm{C}$, and Figure S2F in the Supporting Information) in SAPO11-SFAE.

Although there are several examples of synthesis routes, such as using 3-(trimethoxysilyl)propyl hexadecyl dimethylammonium chloride ${ }^{44}$ or crystallization through repetitive branching $^{45}$ to generate a "house-of-cards"-like morphology for zeolitic aluminosilicates, the only available route to generate nanosheets of SAPOs is through templating by amphiphilic multiamines, as reported by Seo et al. ${ }^{16}$ We demonstrate, for the first time, that the nonclassical growth pathway can be tailored to generate such two-dimensional (2D) assembled architectures for SAPOs, leading to a structure close to an assemblage of mesoporous zeolitic nanosheets. ${ }^{44,45}$ Compared with the AEL SAPO sheets derived with multiamines, ${ }^{16}$ SAPO11-THAM and SAPO-11-SFAE show high crystallinity (XRD pattern) and are thicker $(20-30 \mathrm{~nm}$ vs $2-5 \mathrm{~nm})$, so that a high thermal stability and stronger acidity may be envisaged. Moreover, the preparation is simpler and cost-effective, as the starting materials are common commodities.

For comparison, another series of SAPO-11 was synthesized using an identical batch composition of starting materials, but via a conventional hydrothermal synthesis pathway. No welldeveloped crystalline AEL products were obtained (see Figure S4 in the Supporting Information) for PDDA and MEPB mixed batches. Although SAPO-11 compounds can be fabricated in the presence of THAM and SFAE additives, the aforementioned morphological features are lost (see Figure S5 in the Supporting Information). These results underline the cooperative assembly of additives and crystallites, leading to an orientating crystallization process. In other words, for a successful synthesis, it is indispensable that the growth inhibitors work synergistically with crystalline particulates at the right time.

XRD patterns of SAPO-11-C are shown in Figure 2, for comparison. The diffraction pattern can be attributed to the typical SPAO-11 with AEL structure (JCPDS File No. 42$0428) .{ }^{46,47}$ The absence of additional diffraction peaks indicates that the samples are free of impurities, while the absence of lumps from amorphous raw materials suggests that all of the starting materials have been converted. SEM images for SAPO$11-\mathrm{C}$ are shown in Figures S6A and S6B in the Supporting Information. For comparison, SAPO-11-2.5h was separated and further crystallized in pure $\mathrm{H}_{2} \mathrm{O}$ for an additional $72 \mathrm{~h}$ to achieve complete crystallization in the absence of growth modifiers, the images for which are juxtaposed in Figures S6D and S6E in the Supporting Information. The hydrothermally synthesized SAPO-11-C consists of spherical particulates, whereas the morphological features for a fully crystallized sample resemble those of the parent SAPO-11-2.5h (Figure $\mathrm{S} 6 \mathrm{~F}$ in the Supporting Information). Moreover, the mother liquor for SAPO-11-2.5h was collected after $5 \mathrm{~h}$ of crystallization; it had a very low concentration of inorganics: $\mathrm{Al}, 0.25 \mathrm{mg} / \mathrm{mL}$; Si, $0.093 \mathrm{mg} / \mathrm{mL}$;, $0.41 \mathrm{mg} / \mathrm{mL}$, giving further evidence for the limited dissolution of SAPO-11-2.5h. Since significant dissolution is a prerequisite for the classic atom-by-atom crystal growth, orientated attachment growth becomes the only viable mechanism. The monitored $\mathrm{pH}$ was ca. 7 , the almost-identical morphology showed that the dissolution 

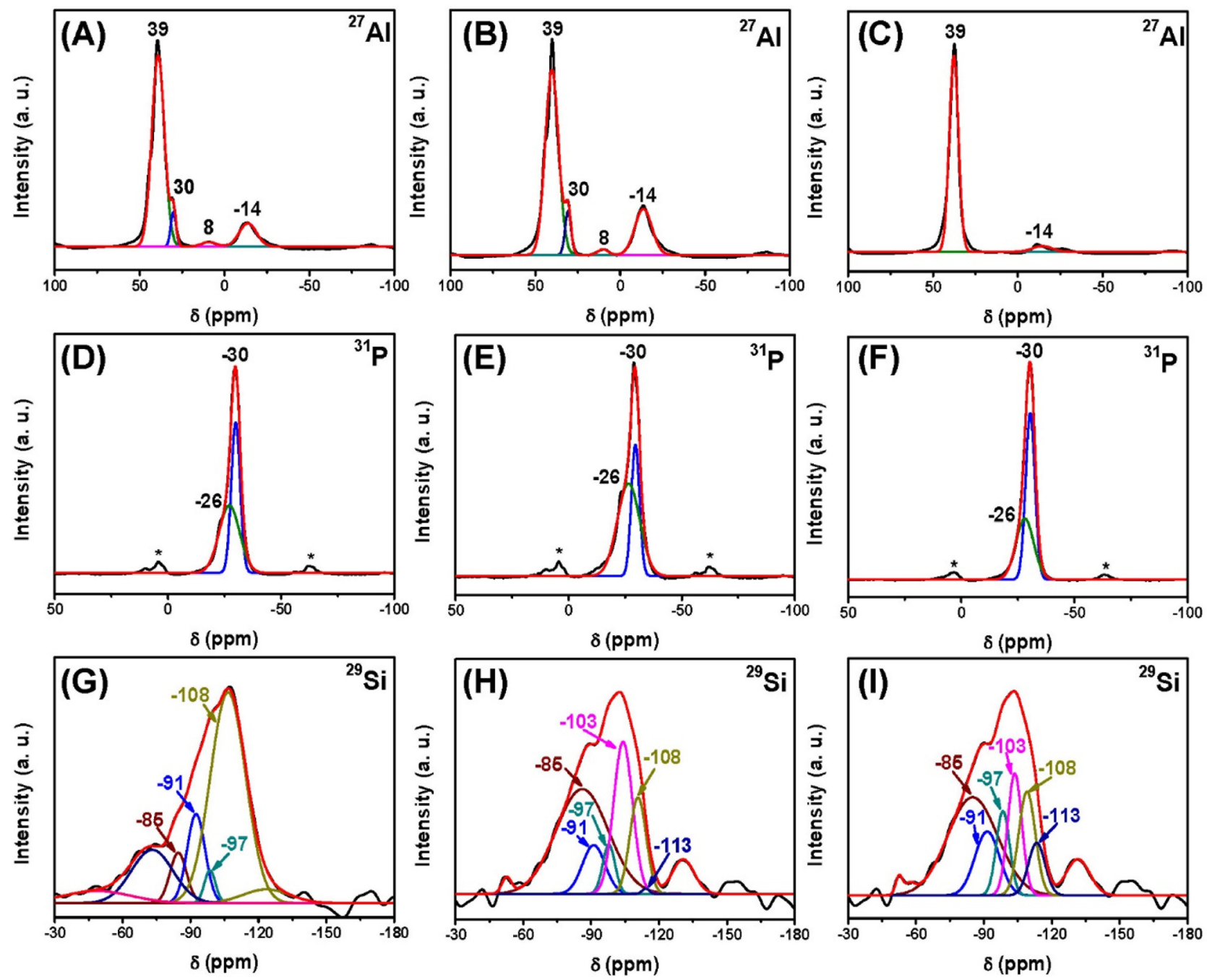

Figure 5. ${ }^{27} \mathrm{Al},{ }^{31} \mathrm{P}$, and ${ }^{29} \mathrm{Si}$ MAS NMR spectra of calcined SAPO-11 samples: (A, D, G) SAPO-11-C, (B, E, H) SAPO-11-PDDA, and (C, F, I) SAPO-11-THAM.

of prefabricated nanocrystallites did not happen, and secondary growth mainly occurred through the orientated attachment growth mechanism. In high-magnification images, it is found that the large SAPO-11-C spherical particulates are composed of irregularly shaped subunits. The TEM images thus obtained for SAPO-11-C are displayed in Figure S6C in the Supporting Information. The primary particles of the building blocks are found to be $200-300 \mathrm{~nm}$ wide, which is close to the size of the building blocks observable in SEM micrographs. The intergrowth of irregularly shaped building blocks for SAPO-11-C is consistent with the $\mathrm{N}_{2}$ physisorption data, since no obvious mesopores have been detected (Figure 4A).

${ }^{27} \mathrm{Al},{ }^{29} \mathrm{Si},{ }^{31} \mathrm{P}$ MAS NMR Spectra. To probe the chemical environment of the components, ${ }^{27} \mathrm{Al},{ }^{29} \mathrm{Si}$, and ${ }^{31} \mathrm{P}$ MAS NMR spectra have been collected. The spectra for representative SAPO-11-C, SAPO-11-PDDA, and SAPO-11-THAM are shown in Figure 5. In ${ }^{27} \mathrm{Al}$ MAS NMR spectra (Figures 5AC), strong peaks appear at 39 and $30 \mathrm{ppm}$ for all three samples, which are assigned to tetrahedrally coordinated framework $\mathrm{Al}$ atoms, and a low peak at $-14 \mathrm{ppm}$, which is assigned to octahedrally coordinated $\mathrm{Al}$ atoms. ${ }^{48,49}$ The weak signal at 8 ppm indicates the presence of a small amount of pentacoordinated $\mathrm{Al}$ atoms. ${ }^{41,48}$ In ${ }^{31} \mathrm{P}$ MAS NMR spectra (Figures 5D-F), the strong peak at $-30 \mathrm{ppm}$ is assigned to tetrahedrally coordinated $\mathrm{P}$ atoms bound to four $\mathrm{Al}$ atoms; meanwhile, the significantly weaker signal at $-26 \mathrm{ppm}$ is attributed to $\mathrm{P}$ atoms that are coordinated to $y$ water molecules, in addition to $4 \mathrm{Al}$ atoms, that is, $\mathrm{P}(\mathrm{OAl})_{x}\left(\mathrm{H}_{2} \mathrm{O}\right)_{y}$ with $x=4,{ }^{41,50}$ or which are coordinated to $y$ water molecules instead of $\mathrm{Al}$ atoms, that is, $\mathrm{P}(\mathrm{OAl})_{x}\left(\mathrm{H}_{2} \mathrm{O}\right)_{y}$ with $x=4-y{ }^{41,51}$ These observations demonstrate that both $\mathrm{Al}$ and $\mathrm{P}$ are incorporated into the framework of SAPO-11. No significant extra-framework species have been found in SAPO-11-PDDA and SAPO-11-THAM.

In the formation of acid sites in the otherwise electronneutral AlPO framework, $\mathrm{Si}$ substitution occurs via two mechanisms: SM2 and SM3. ${ }^{19,41}$ In the SM2 type, one Si substitutes a $\mathrm{P}$ atom to form a $\mathrm{Si}(\mathrm{OSi}, 4 \mathrm{Al})$ site and gives rise to a weak Brønsted acid site, whereby a proton charge balances the negatively charged framework. In the SM3 type, two Si atoms substitute an $\mathrm{Al}+\mathrm{P}$ pair. Since a $\mathrm{Si}-\mathrm{O}-\mathrm{P}$ bond is strictly prohibited, ${ }^{52}$ because of the high energy cost to form it, the SM3 mechanism only occurs alongside the SM2 mechanism to form silica islands. In the inner part of a silica island, only nonacidic $\mathrm{Si}(4 \mathrm{Si}, 0 \mathrm{Al})$ sites form, whereas $\mathrm{Si}(4-n \mathrm{Si}, n \mathrm{Al})(n=$ 1-4) forms at the border of a silica island to produce strong acid sites. The chemical shifts of these sites can be clearly discriminated by deconvoluting the ${ }^{29} \mathrm{Si}$ MAS NMR envelopes (Figures $5 \mathrm{G}-\mathrm{I})$, as signals related to $\mathrm{Si}(0 \mathrm{Si}, 4 \mathrm{Al}), \mathrm{Si}(1 \mathrm{Si}, 3 \mathrm{Al})$, 
Table 2. Deconvolution Results of the ${ }^{29}$ Si MAS NMR Spectra of SAPO-11, Based on the Normalized Peak Areas of the Different Si Species

\begin{tabular}{|c|c|c|c|c|c|c|}
\hline \multirow[b]{2}{*}{ sample } & \multicolumn{6}{|c|}{ Deconvolution (\%) } \\
\hline & $\mathrm{Si}(\mathrm{OH})$ or $\mathrm{Si}(\mathrm{OH})_{2},{ }^{a}-85 \mathrm{ppm}$ & $\mathrm{Si}(4 \mathrm{Al}),-91 \mathrm{ppm}$ & $\mathrm{Si}(3 \mathrm{Al}),-97 \mathrm{ppm}$ & $\mathrm{Si}(2 \mathrm{Al}),-103 \mathrm{ppm}$ & $\mathrm{Si}(1 \mathrm{Al}),-108 \mathrm{ppm}$ & $\mathrm{Si}(0 \mathrm{Al}),-113 \mathrm{ppm}$ \\
\hline SAPO-11-C & 8.1 & 15.6 & 3.6 & 0 & 72.7 & 0 \\
\hline SAPO-11-PDDA & 46.9 & 9.5 & 5.2 & 24.7 & 13.4 & 0.3 \\
\hline SAPO-11-THAM & 41.3 & 13.2 & 9.4 & 14.4 & 14.4 & 7.3 \\
\hline
\end{tabular}

Table 3. Py-IR and 2,4,6-Collidine-IR Measurements of Acidity for SAPO-11 Derived from Different Routes

\begin{tabular}{|c|c|c|c|c|c|c|c|c|}
\hline \multirow[b]{3}{*}{ sample } & \multicolumn{6}{|c|}{ Acidity $\left(\mu \mathrm{mol} \mathrm{g}^{-1}\right)$} & & \\
\hline & \multicolumn{2}{|c|}{ Brønsted Acidity $^{a}\left(1545 \mathrm{~cm}^{-1}\right)$} & \multicolumn{2}{|c|}{ Lewis Acidity $^{a}\left(1455 \mathrm{~cm}^{-1}\right)$} & \multicolumn{2}{|c|}{$\begin{array}{c}\text { External Brønsted Acidity } \\
\left(1638 \mathrm{~cm}^{-1}\right)\end{array}$} & \multicolumn{2}{|c|}{ Accessibility, $\chi^{c}(\%)$} \\
\hline & $473 \mathrm{~K}$ & $623 \mathrm{~K}$ & $473 \mathrm{~K}$ & $623 \mathrm{~K}$ & $473 \mathrm{~K}$ & $623 \mathrm{~K}$ & $473 \mathrm{~K}$ & $623 \mathrm{~K}$ \\
\hline SAPO-11-C & 87 & 46 & 40 & 28 & 40 & 28 & 46.0 & 60.9 \\
\hline SAPO-11-PDDA & 95 & 38 & 59 & 31 & 36 & 17 & 37.9 & 44.7 \\
\hline SAPO-11-MEPB & 58 & 39 & 44 & 27 & 31 & 20 & 53.4 & 51.3 \\
\hline SAPO-11-THAM & 130 & 44 & 35 & 28 & 34 & 17 & 26.2 & 38.6 \\
\hline SAPO-11-SFAE & 122 & 32 & 30 & 24 & 32 & 11 & 26.2 & 34.4 \\
\hline
\end{tabular}

${ }^{a}$ The numbers of Brønsted acid sites and Lewis acid sites were calculated from the Py-IR band area located at 1545 and $1455 \mathrm{~cm}^{-1}$, respectively. ${ }^{b}$ The number of external Brønsted acid sites was calculated from the 2,4,6-collidine-IR band area located at $1638 \mathrm{~cm}^{-1}$. ${ }^{c}$ Defined as the ratio of the number of external Brønsted acid sites to the total number of Brønsted acid sites.

$\mathrm{Si}(2 \mathrm{Si}, 2 \mathrm{Al}), \mathrm{Si}(3 \mathrm{Si}, 1 \mathrm{Al})$ and $\mathrm{Si}(4 \mathrm{Si}, 0 \mathrm{Al})$ appear at $-91 \mathrm{ppm}$, $-97 \mathrm{ppm},-103 \mathrm{ppm},-108 \mathrm{ppm}$ and $-113 \mathrm{ppm}$, respectively. ${ }^{53}$ Meanwhile, $(\mathrm{AlO})_{3} \mathrm{Si}(\mathrm{OH})$ or $(\mathrm{SiO})(\mathrm{AlO}) \mathrm{Si}$ $(\mathrm{OH})_{2}$ sites $(-85 \mathrm{ppm})$, rich in silanol groups, can be identified, which are found to increase in content for hierarchical SAPO-11-PDDA and SAPO-11-THAM, corresponding to increasing external surface areas. This may arise from the strong interaction between growth modifiers and the SAPO-11 surface, formation of which inhibits the growth of SAPO-11 to its full size. The $-85 \mathrm{ppm}$ signal has been attributed to the formation of silica-alumina (SA)-type substitution, ${ }^{42,54-56}$ which, however, is not supported by the ${ }^{27} \mathrm{Al}$ and ${ }^{31} \mathrm{P}$ NMR spectra. Recent ${ }^{1} \mathrm{H}-{ }^{29} \mathrm{Si} \mathrm{CP}$ NMR studies show that the $-85 \mathrm{ppm}$ is associated with a nearby proton site. $^{57}$ A quantitative analysis (Table 2) of SAPO-11-C shows that there is far less $(\mathrm{AlO})_{3} \mathrm{Si}(\mathrm{OH})$ or $(\mathrm{SiO})(\mathrm{AlO}) \mathrm{Si}(\mathrm{OH})_{2}$ than in the other two samples, indicating that interaction between additives and nanocrystalline building blocks has incurred some surface-defective sites. The spectra also manifest that there is an insignificant contribution from $\mathrm{Si}(4 \mathrm{Si}, 0 \mathrm{Al})$, indicating the formation of small silica islands. The concentration of weakly acidic $\mathrm{Si}(0 \mathrm{Si}, 4 \mathrm{Al})$ sites is comparable for SAPO-11-C (15.6\%) and SAPO-11-THAM (13.2\%), but lower in SAPO-11-PDDA (9.5\%). Because small silica islands are rich in $\mathrm{Si}(4-n \mathrm{Si}, n \mathrm{Al})$ sites but have less $\mathrm{Si}(4 \mathrm{Si}, 0 \mathrm{Al})$ sites, the SAPO-11-PDDA and SAPO-11-THAM have a $\mathrm{Si}$ dispersion that is comparable to SAPO-11-C. ${ }^{46}$ Overall, the prepared hierarchical SAPO-11 compounds possess improved $\mathrm{Si}$ dispersion and small Si patches, i.e., the introduction of a hierarchical structure by the assembly of prefabricated particulates does not significantly alter the structural integrity or the manner of $\mathrm{Si}$ incorporation.

Acidity Measurements. The types and strengths of acid sites has been determined by infrared spectroscopy, using pyridine (Py-IR) as a probe base molecule at 473 and $623 \mathrm{~K}$, respectively. Pyridine can access acid sites located inside the micropores of an AEL crystal and can thus be applied to determine the total number of acid sites. ${ }^{58}$ The Py-IR spectra for the five samples are shown in Figure S7 in the Supporting Information. The bands at 1545 and $1455 \mathrm{~cm}^{-1}$ can be assigned to pyridine adsorbed on Brønsted (B) and Lewis (L) acid sites, respectively. ${ }^{59,60}$ The peak at $1490 \mathrm{~cm}^{-1}$ is a combined result from both sites. ${ }^{61}$ The areas of the peaks decrease gradually when the outgassing temperature rises from $473 \mathrm{~K}$ to $623 \mathrm{~K}$, as a consequence of the desorption of weakly adsorbed pyridine from hydroxyl groups associated with acid sites. ${ }^{58}$ Table 3 presents the quantitative results of the types and distribution of acid sites for these samples, derived from the Py-IR spectra. The numbers of catalytically relevant medium and strong Brønsted acid sites for SAPO-11-PDDA, SAPO-11-MEPB, SAPO-11-THAM, and SAPO-11-SFAE (measured at $623 \mathrm{~K}$ ) are $38,39,44$, and $32 \mu \mathrm{mol} \mathrm{Py} \mathrm{g}^{-1}$, respectively, which are smaller than that of SAPO-11-C (46 $\mu$ mol Py $\left.\mathrm{g}^{-1}\right)$. The preservation of strong acidity hinges on $\mathrm{Si}$ incorporation into the AlPO framework and crystallographic ordering of the samples; these numbers confirm that acidity has been preserved by the precrystallization process in our synthesis. These results also agree with the aforementioned NMR measurements, corroborating insignificant acid strength reduction for hierarchical architectures.

2,4,6-Collidine-IR (Coll, $7.4 \AA$ in diameter) was employed to evaluate acid sites situated exclusively on the external surface, as this probe molecule cannot access the micropores of the AEL structure. $^{62}$ This means that the adsorbed quantities measured by Coll-IR are associated purely with acid sites on the surface of mesopores or macropores that are accessible via the exterior surface of such SAPO-11 crystals. $^{22}$ Gilson et al. ${ }^{62,63}$ associated the vibrations at $1638 \mathrm{~cm}^{-1}$ to a Coll molecule interacting with Brønsted acid sites. The detailed results are shown in Figure S8 in the Supporting Information and in Table 3. The ratio of the number of external Brønsted acid sites to the total number of Brønsted acid sites (defined as $\chi$ ) is $60.9 \%, 44.7 \%, 51.3 \%$, $38.6 \%$, and $34.4 \%$ at $623 \mathrm{~K}$ for SAPO-11-C, SAPO-11-PDDA, SAPO-11-MEPB, SAPO-11-THAM, and SAPO-11-SFAE, respectively. By comparing the NMR results with the measurements of the acidity via IR spectroscopy, it is deduced 
that more Brønsted acid sites are distributed on the external surface, since additives interact strongly with the external surface, as found by the hand-in-hand increase in the number of defective sites ( $-85 \mathrm{ppm}^{29} \mathrm{Si}$ NMR peak) with external acidity. The distribution of acid sites is important, because the catalytic behavior of external acid sites may compromise shape selectivity. $^{20}$

Catalytic Assessment in $n$-Heptane Hydroisomerization. The five samples were loaded with 0.5 wt $\% \mathrm{Pt}$, and their catalytic properties were assessed via the hydroisomerization of $n$-heptane. Such a $\mathrm{Pt} / \mathrm{H}^{+}$ratio (Table 1 ) is sufficient to ensure that the dehydrogenation of $n$-heptane is not the ratedetermining step, and the performance is solely determined by SAPO-11. ${ }^{10,11,29,64}$ The products mainly consist of MB (e.g., 2- or 3- methyl hexane) and DB (e.g., 2,3- or 2,4-dimethyl pentane) isomers, together with cracking products (Table S1). A low selectivity to tribranched (TB, e.g., 2,2,3-trimethylbutane) isomers was only detected for SAPO-11-PDDA and SAPO-11-MEPB, because of a large fraction of acid sites on their external surfaces, as TB isomers are too bulky to enter the micropores. ${ }^{10}$ The biased distribution of acid sites on the external surface for the two samples may be associated with the Coulombic interaction between cationic additives and negatively charged nanoscale building blocks. The latter may induce a larger number of negatively charged Si centers to be located on the external surface, thus balancing the positive charge of the additives. The catalytic activity increases, leading to faster rates for Pt/SAPO-11-PDDA, Pt/SAPO-11-THAM, and Pt/SAPO-11-SFAE than for Pt/SAPO-11-C (for conversion versus temperature; see Figure S9A in the Supporting Information). Meanwhile, the selectivity to $\mathrm{MB}$ isomers decreases as the temperature increases (Figure 6C), whereas the selectivity to $\mathrm{DB}$ isomers first increases with temperature and then peaks with a broad maximum, before decreasing with further increases in temperature (Figure 6D). Isomer selectivity for all hierarchical samples outperforms the conventional $\mathrm{Pt} /$ SAPO-11-C, and Pt/SAPO-11-PDDA shows an outstanding total isomer selectivity (Figure S9B in the Supporting Information). All samples are stable for a time-on-stream test of at least $51 \mathrm{~h}$, which is the length of the experiments (see Figure S10 in the Supporting Information). The stability is attributed to the presence of $\mathrm{H}_{2}$, which suppresses coke formation under isomerization conditions, ${ }^{65,66}$ which also demonstrates that the SAPO-11 structures are preserved during the tests. When plotting the conversion vs isomer yield in Figure 6A, it is manifested that the overall yield increases with $n$-heptane conversion for all samples until a maximum is attained, and the divergence is caused by cracking reactions of DBs. The maximum yield of isomers ranks in the following order: Pt/SAPO-11-PDDA > Pt/SAPO-11-SFAE > Pt/SAPO11-THAM > Pt/SAPO-11-MEPB > Pt/SAPO-11-C. The highest yield of isomers is found to be $79 \%$ for Pt/SAPO-11PDDA. It is noteworthy that this value is much higher than the $51 \%$ yield that has been reported by Seo et al. ${ }^{16}$ for a hierarchical ATO SAPO, and this value is the highest yield of isomers for $n$-heptane hydroisomerization ever reported (see Table S2). The yield of isomers is found to be dependent on reaction temperature for each catalyst (Figure 6B), which significantly influences the product distribution as well.

Kinetic Studies. To shed light on the effect of diffusion toward the observed catalytic behavior, kinetic measurements were conducted to determine the activation energies. External transport limitations during the kinetic studies have been
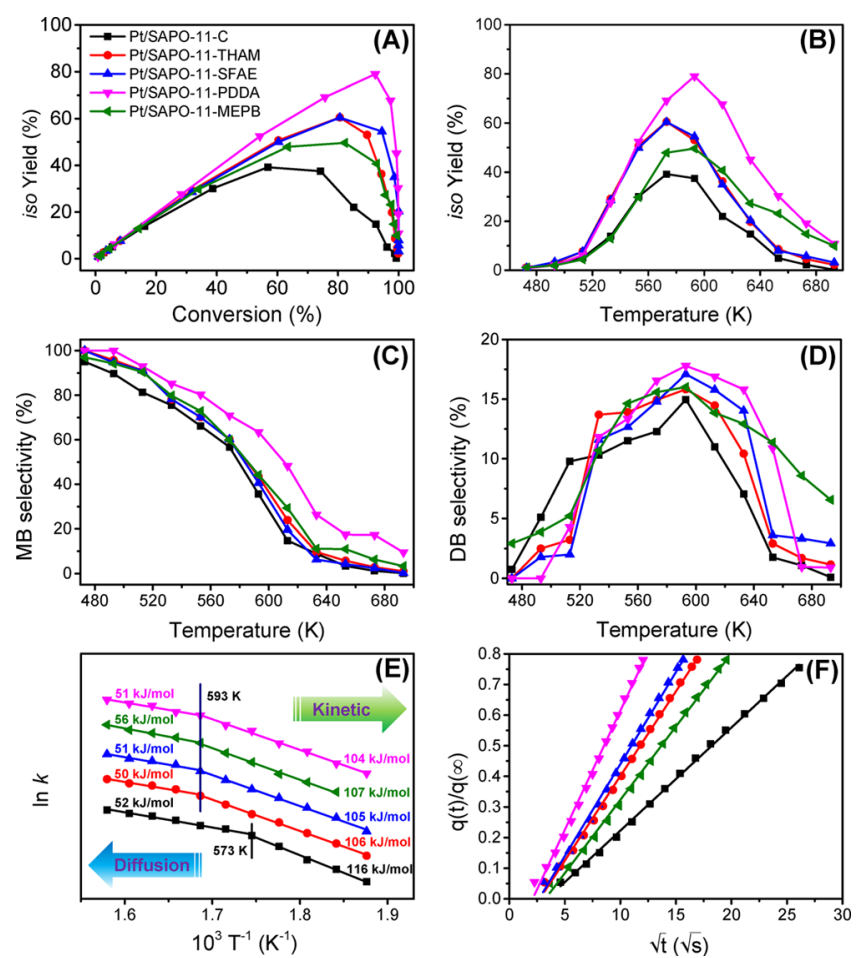

Figure 6. (A) Isomer yields versus reactant conversion and (B) isomer yields versus temperature, $(\mathrm{C})$ selectivity to monobranched isomers, and (D) dibranched isomers versus temperature in $n$-heptane hydroisomerization. Arrhenius plot of (E) the n-heptane hydroconversion (shifted upward by a factor of $0.9,1.9,4.3$, and 3.4 for Pt/ SAPO-11-THAM, Pt/SAPO-11-SFAE, Pt/SAPO-11-PDDA, and Pt/ SAPO-11-MEPB, respectively) and (F) adsorption rates of 2methylhexane in the calcined SAPO-11 compounds.

precluded by controlling the flow rate, whereas internal diffusion limitations cannot be ruled out. Therefore, the measured activation energy in an Arrhenius plot reflects the lumped effect from diffusion and intrinsic kinetics, as displayed in Figure 6E.

For Pt/SAPO-11-C, an activation energy of $116 \mathrm{~kJ} \mathrm{~mol}^{-1}$ is deduced for reactions that were performed at $<573 \mathrm{~K}$, but a lower apparent activation energy of ca. $52 \mathrm{~kJ} \mathrm{~mol}^{-1}$ (approximately half of the value observed at lower temperatures) was obtained for higher temperatures, which confirms the observations of Höchtl et al. ${ }^{10}$ This means that the rate of the isomerization process is controlled by intrinsic reaction at low temperatures for SAPO-11-C, but by diffusion at temperatures higher than $573 \mathrm{~K}$ (at a conversion of $56.9 \%$ ). For hierarchical samples, the same trend can be observed, but the transition temperature has increased to $593 \mathrm{~K}$. At the same temperature, much higher conversions of $n$-heptane have been obtained $(92.3 \%, 82.3 \%, 89.6 \%$ and $94.5 \%$ for SAPO-11PDDA, SAPO-11-MEPB, SAPO-11-THAM, and SAPO-11SFAE, respectively). By correlating the transition temperature from the intrinsic reaction-controlled regime to the diffusioncontrolled regime, with the corresponding selectivity toward various isomers (Figures $6 \mathrm{~B}$ and $6 \mathrm{D}$ ), it is instructive to learn that the maximum selectivity for DBs, as well as the overall yield for isomers (Figure 6B) always appears in the region close to the transition temperature. This relationship guides us to understand that the key reason for the loss in isomerization selectivity is associated with entering the diffusion-controlled regime for the catalyst. This makes sense, as the isomerization 
reaction predominates within the intrinsic reaction-controlled regime, while cracking side reactions contribute significantly to selectivity only when diffusion limitations become important.

Diffusivity Measurement. To quantify the extent to which the diffusion resistance has been mitigated by various hierarchical architectures, diffusion measurements were carried out with 2-methylhexane as a representative adsorbate. 2Methylhexane is also one of the dominant primary isomerization products. Diffusion is crucial, because it determines the residence time inside the micropores, and a longer residence time will lead to a higher cracking probability of isomerized products. The effective diffusivity was estimated using Fick's second law, which expresses the concentration variation of molecules inside a porous slab (assumed infinitely extended in the transverse directions), as a function of exposure time:

$$
\frac{\partial C}{\partial t}=D_{\text {eff }}\left(\frac{\partial^{2} C}{\partial x^{2}}\right)
$$

where $C$ is the concentration of 2-methylhexane inside the particle, $t$ the time, $D_{\text {eff }}$ the diffusivity, and $x$ the distance, measured from the center of a slab.

At the beginning of the adsorption process in a slablike crystal, the solution of this equation can be well approximated by $^{20,67}$

$$
\frac{q(t)}{q(\infty)}=\frac{2}{\sqrt{\pi}} \sqrt{\frac{D_{\text {eff }}}{L^{2}}} \sqrt{t}
$$

where $q(t) / q(\infty)$ is the normalized hydrocarbon uptake and $L$ is the characteristic diffusion length. The slab model is pertinent in this case, because it is suitable for anisotropic diffusion along the unidimensional pores along the $c$-axis of the AEL structure. Figure $6 \mathrm{~F}$ shows a linear relationship between $q(t) / q(\infty)$ and $t^{1 / 2}$, in agreement with this hypothesis. Values for $D_{\text {eff }} / L^{2}$, calculated from the slopes, are summarized in Table 4. Fundamentally, $D_{\text {eff }}$ is determined by the microporous

Table 4. Calculated Values of the Inverse of the Diffusion Time Constant $\left(D / L^{2}\right)$, Based on Figure $6 \mathrm{~F}$

\begin{tabular}{lc}
\multicolumn{1}{c}{ sample } & $D / L^{2}\left(\mathrm{~s}^{-1}\right)$ \\
SAPO-11-C & $0.89 \times 10^{-3}$ \\
SAPO-11-THAM & $2.40 \times 10^{-3}$ \\
SAPO-11-SFAE & $2.84 \times 10^{-3}$ \\
SAPO-11-PDDA & $4.75 \times 10^{-3}$ \\
SAPO-11-MEPB & $1.80 \times 10^{-3}$ \\
\hline
\end{tabular}

structure, which is specific to the AEL topology and should be identical for all samples. ${ }^{67}$ Therefore, the variation in $D_{\text {eff }} / L^{2}$ value is representative for a change in mass transfer rate, which increases with decreasing $L$, as a result of diffusion length shortening by the introduction of auxiliary porosity. ${ }^{19,20}$ Nevertheless, it is noteworthy that the quantitation of diffusion is based on a few simplifications, including the assumption that the individual microporous particles have the same intrinsic transport properties, which may no longer hold for systems with a prominent influence from surface barriers to permeation. ${ }^{68}$ The fact that the straight lines shown in Figure $6 \mathrm{D}$ do not pass through the origin may be an indication that mass exchange is also affected by surface permeability. ${ }^{68}$ The results indicate that the $D_{\text {eff }} / L^{2}$ values increase in the following order: SAPO-11-C < SAPO-11-MEPB < SAPO-11-THAM < SAPO-11-SFAE < SAPO-11-PDDA. Such an order offers a direct measure for the increase in mass transfer rate for hierarchical architectures, and the order coincides with the selectivity for isomer yields, despite their difference in acidity, because of diffusion control. Note that SAPO-11-PDDA has remarkable diffusion properties that agree well with the small crystallite size and pore-network connectivity, as well as high surface area.

General Discussion on Architecture-Catalysis Relationship. $n$-Heptane hydroisomerization, similar to that observed for other $n$-paraffins, occurs via dehydrogenation on Pt sites to form $n$-alkenes (olefins), which proceeds via isomerization on Brønsted acid sites of SAPO-11 to afford iso-alkenes, before being rehydrogenated on $\mathrm{Pt}$ sites to isoparaffins. ${ }^{12,69}$ The dehydrogenation and rehydrogenation steps reach equilibrium quickly, and, therefore, the overall reaction is controlled by the isomerization process. $n$-Heptane isomerization is a sequential reaction, with $\mathrm{MB}$ isomers being the primary products and $\mathrm{DB}$ isomers being the secondary products. ${ }^{12}$ The unwanted cracking products mainly stem from cracking of $\mathrm{DB}$ products, as $n$-heptane or $\mathrm{MB}$ isomers have a much smaller cracking probability. ${ }^{10,12} \mathrm{~TB}$ isomers are mainly formed for the two samples that have more strong acid sites located on their external surface, as they are mainly produced by unrestricted isomerization. ${ }^{10,12}$

The increase in isomerization reaction rate with increasing temperature for $n$-heptane or MB isomers results in an increase in concentration of isomers inside the micropores. ${ }^{10}$ Simultaneously, the cracking rate also increases with temperature, but insignificantly before the entire process enters the diffusioncontrolled regime. This also explains why the measured cracking rate is low in kinetic studies performed at low conversion and temperature, ${ }^{10}$ and low selectivity to cracking reactions was always detected under such conditions. ${ }^{10,19}$ In the diffusion-controlled regime, reversible isomerization reactions between $n$-heptane and the corresponding isomers reach thermodynamic equilibrium as the rate-determining step is diffusion, and reaction kinetics become unimportant. The drastic increase in cracking rate is not a result of opening a new reaction channel at high temperatures, but stems from the accumulation of thermodynamically favored DB isomers in the microchannels of SAPO-11, because of sluggish diffusion, as shown in Scheme 2. DB isomers can either desorb from the micropores of the AEL structure or undergo cracking before desorption, but are unlikely to be significantly readsorbed inside the micropores from the gas phase, because of their bulky size,

Scheme 2. Reaction Scheme and Reaction-Diffusion Interplay in $n$-Heptane Hydroisomerization on Pt/SAPO-11

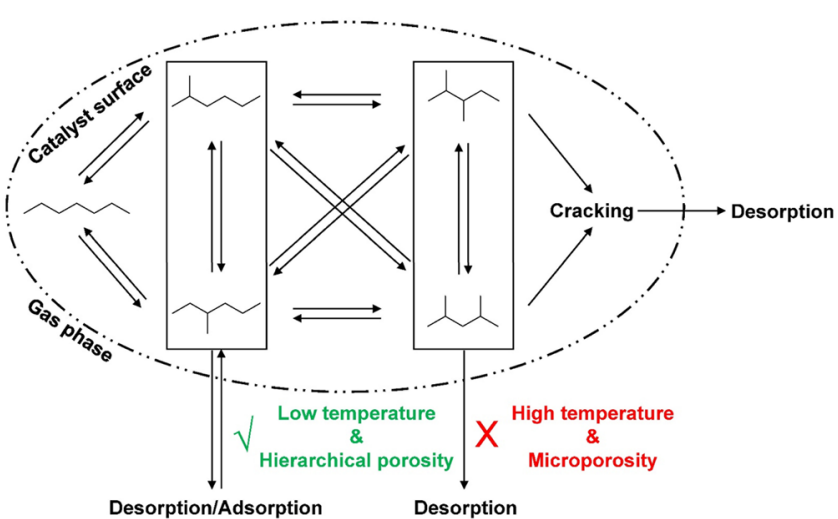


so that they are mainly adsorbed on pore-mouth sites. ${ }^{10}$ As cracking of $\mathrm{DB}$ isomers is also believed to be an irreversible process, the slow diffusion of $\mathrm{DB}$ isomers accelerates the cracking reaction considerably upon entering the diffusioncontrolled regime at elevated temperatures. Such an inference is advocated by the similar distribution of cracking products in both regimes (see Figure S11 in the Supporting Information), as a high concentration of $\mathrm{DB}$ isomers will not alter the mechanism or kinetic characteristics, but only increase the cracking rate. The introduction of secondary porosity leads to a shortened residence time for primary $\mathrm{MB}$ isomers and consecutive $\mathrm{DB}$ isomers, and, as a result, a higher isomer selectivity is detected in the effluent gas. A short residence time for $\mathrm{MB}$ isomers also reduces their concentration inside the micropores and slows the transformation to DB isomers; consequently, a reduction in concentration of DB isomers at increased conversion can be anticipated.

Combining these mechanistic insights with the kinetic and diffusivity measurements, it is reasonable to conclude that a preferred isomerization catalyst should have two properties. First, an architecture that favors mass transport, but with optimized, sufficiently short residence time. Architectures featuring a small primary crystal size, with a hierarchical macropore/mesopore/micropore network, together with a large surface area are preferred, because such factors contribute to enhanced fast mass transport for isomers. The hierarchical structure also expands the intrinsic reaction-controlled regime. Second, strong Brønsted acidity is desirable, as it drives catalysis at lowered temperatures (based on the Arrhenius relationship), keeping the process operating within, but just on the edge of, a kinetically controlled regime that disfavors cracking. Such an effect of subtly optimized hierarchical architecture over catalytic performance has not previously been disclosed, to the best of our knowledge. As n-paraffin isomerization is representative for a family of analogous catalytic processes, we anticipate that the relationship is of broader validity for other processes that are dependent on the interplay of diffusion and reaction, with consecutive steps that require the right balance to achieve high selectivity.

\section{CONCLUSIONS}

In summary, for the first time, we demonstrated that the hierarchical architecture of SAPOs can be manipulated via an orientated assembly synthesis process, paving the road toward an inexpensive route that warrants mesoscale structural control, without losing crystallinity and acidity. The advantages of the synthesis include (1) fast nucleation in DGC, ensuring small primary crystal size and narrow size distribution control; (2) orientated assembly of build blocks, facilitating hierarchical structure manipulation; (3) pore connectivity, which can be determined by growth modifiers; and (4) tunable dimension and orientation for the assembled architectures. This finding opens up new opportunities for improving the catalytic properties for SAPOs through morphological and porestructural control. The preferred architecture for $n$-heptane hydroisomerization should possess a small primary crystallite size and penetrating auxiliary mesoporosity/macroporosity that facilitates the mass transfer of isomers, especially for DB isomers, as consistently shown by catalytic assessments, kinetic studies, and diffusivity measurements. Future catalyst design should be directed to enhance both acid strength and mass transfer properties of SAPOs or zeolites. The influences of the hierarchical structure on hydroisomerization selectivity are found to be 2-fold: widening of the intrinsic reaction-controlled regime, in addition to enhanced diffusivity that lowers the cracking probability. As far as operation conditions are concerned, hydroisomerization catalyzed by zeotype materials, generally should only be operated within the intrinsic reactioncontrolled regime, in order to maintain a high isomerization selectivity.

\section{ASSOCIATED CONTENT}

Supporting Information

The Supporting Information is available free of charge on the ACS Publications website at DOI: 10.1021/acscatal.7b01646.

SEM micrographs of time-dependent tracking for crystallization of SAPO-11 in a DGC synthesis; mercury intrusion-extrusion curves and the corresponding pore size distribution of SAPO-11 compounds; high-resolution TEM micrographs for SAPO-11-SFAE; XRD patterns for SAPO-11 synthesized from a conventional hydrothermal route in the presence of PDDA and MEPB; XRD patterns and SEM micrographs for SAPO11 synthesized from a conventional hydrothermal route in the presence of THAM and SFAE; SEM and TEM micrographs of SAPO-11-C; Fourier transform infrared (FT-IR) spectra of pyridine (Py) adsorbed on synthesized SAPO-11 compounds at 473 and $623 \mathrm{~K}$; FT-IR spectra of 2,4,6-collidine adsorbed on synthesized SAPO11 compounds at different evacuation temperatures (473 and $623 \mathrm{~K}$ ); conversion of $n$-heptane versus reaction temperatures and total isomerization selectivity versus temperature for all catalysts; time-on-stream catalytic stability and isomer yield of Pt/SAPO-11 compounds for $n$-heptane isomerization; selectivity of cracking for Pt/ SAPO-11 compounds at 533 and $633 \mathrm{~K}$; the product distribution for $n$-heptane hydroisomerization catalyzed by $\mathrm{Pt} / \mathrm{SAPO}-11$ compounds at maximum yield; and reported data for the catalysts on the hydroisomerization of $n$-heptane (PDF)

\section{AUTHOR INFORMATION}

\section{Corresponding Authors}

*E-mail: kakezhu@ecust.edu.cn (K. Zhu).

*E-mail: m.coppens@ucl.ac.uk (M.-O. Coppens).

ORCID $\odot$

Marc-Olivier Coppens: 0000-0002-1810-2537

Notes

The authors declare no competing financial interest.

\section{ACKNOWLEDGMENTS}

K.Z. is grateful for the financial support from National Natural Science Foundation of China (No. 21576082). X.Z. is sponsored by the National Natural Science Foundation of China (No. U1162112), Fundamental Research Funds for the Central Universities (No. WB 222201718003) and Financial Support from Sinopec Group (Contract No. 415063). M.-O.C. is supported by the EPSRC "Frontier Engineering" Centre for Nature Inspired Engineering (No. EP/K038656/1) and the UK Catalysis Hub (No. EP/K014706/1).

\section{REFERENCES}

(1) De Yoreo, J. J.; Gilbert, P. U. P. A.; Sommerdijk, N. A. J. M.; Penn, R. L.; Whitelam, S.; Joester, D.; Zhang, H.; Rimer, J. D.; 
Navrotsky, A.; Banfield, J. F.; Wallace, A. F.; Michel, F. M.; Meldrum,

F. C.; Coelfen, H.; Dove, P. M. Science 2015, 349, aaa6760.

(2) Lupulescu, A. I.; Rimer, J. D. Science 2014, 344, 729-732.

(3) Chen, X.; Qiao, M.; Xie, S.; Fan, K.; Zhou, W.; He, H. J. Am. Chem. Soc. 2007, 129, 13305-13312.

(4) Moeller, K.; Yilmaz, B.; Mueller, U.; Bein, T. Chem.-Eur. J. 2012, 18, 7671-7674.

(5) Chen, J.; Hua, W.; Xiao, Y.; Huo, Q.; Zhu, K.; Zhou, X. Chem.Eur. J. 2014, 20, 14744-14755.

(6) Greer, H.; Wheatley, P. S.; Ashbrook, S. E.; Morris, R. E.; Zhou, W. J. Am. Chem. Soc. 2009, 131, 17986-17992.

(7) Kumar, M.; Li, R.; Rimer, J. D. Chem. Mater. 2016, 28, 17141727.

(8) Kumar, M.; Luo, H.; Roman-Leshkov, Y.; Rimer, J. D. J. Am. Chem. Soc. 2015, 137, 13007-13017.

(9) Gao, B. B.; Tian, P.; Li, M. R.; Yang, M.; Qiao, Y. Y.; Wang, L. Y.; Xu, S. T.; Liu, Z. M. J. Mater. Chem. A 2015, 3, 7741-7749.

(10) Höchtl, M.; Jentys, A.; Vinek, H. J. Catal. 2000, 190, 419-432.

(11) Maesen, T. L. M.; Schenk, M.; Vlugt, T. J. H.; Jonge, J. P. d.; Smit, B. J. Catal. 1999, 188, 403-412.

(12) Campelo, J. M.; Lafont, F.; Marinas, J. M. J. Catal. 1995, 156, $11-18$.

(13) Sastre, G.; Chica, A.; Corma, A. J. Catal. 2000, 195, 227-236.

(14) Raybaud, P.; Patrigeon, A.; Toulhoat, H. J. Catal. 2001, 197, 98-112.

(15) Höchtl, M.; Jentys, A.; Vinek, H. Catal. Today 2001, 65, 171177.

(16) Seo, Y.; Lee, S.; Jo, C.; Ryoo, R. J. Am. Chem. Soc. 2013, 135, $8806-8809$.

(17) Fan, Y.; Xiao, H.; Shi, G.; Liu, H.; Bao, X. J. Catal. 2012, 285, 251-259.

(18) Liu, Y.; Qu, W.; Chang, W.; Pan, S.; Tian, Z.; Meng, X.; Rigutto, M.; Made, A. v. d.; Zhao, L.; Zheng, X.; Xiao, F.-S. J. Colloid Interface Sci. 2014, 418, 193-199.

(19) Blasco, T.; Chica, A.; Corma, A.; Murphy, W. J.; AgúndezRodríguez, J.; Pérez-Pariente, J. J. Catal. 2006, 242, 153-161.

(20) Kim, M. Y.; Lee, K.; Choi, M. J. Catal. 2014, 319, 232-238.

(21) Verboekend, D.; Milina, M.; Pérez-Ramírez, J. Chem. Mater. 2014, 26, 4552-4562.

(22) Milina, M.; Mitchell, S.; Crivelli, P.; Cooke, D.; Pérez-Ramírez, J. Nat. Commun. 2014, 5, 3922.

(23) Kortunov, P.; Vasenkov, S.; Karger, J.; Valiullin, R.; Gottschalk, P.; Fe Elia, M.; Perez, M.; Stocker, M.; Drescher, B.; McElhiney, G.; Berger, C.; Glaser, R.; Weitkamp, J. J. Am. Chem. Soc. 2005, 127, 13055-13059.

(24) Wang, J.; Groen, J. C.; Yue, W.; Zhou, W.; Coppens, M.-O. Chem. Commun. 2007, 4653-4655.

(25) Wang, J.; Groen, J. C.; Yue, W.; Zhou, W.; Coppens, M.-O. J. Mater. Chem. 2008, 18, 468-474.

(26) Gheorghiu, S.; Coppens, M.-O. AIChE J. 2004, 50, 812-820.

(27) Fan, J.; Yu, C.; Gao, F.; Lei, J.; Tian, B.; Wang, L.; Luo, Q.; Tu,

B.; Zhou, W.; Zhao, D. Angew. Chem., Int. Ed. 2003, 42, 3146-3150.

(28) Sakamoto, Y.; Kaneda, M.; Terasaki, O.; Zhao, D. Y.; Kim, J. M.; Stucky, G.; Shin, H. J.; Ryoo, R. Nature 2000, 408, 449-453.

(29) Guisnet, M. Catal. Today 2013, 218-219, 123-134.

(30) Onfroy, T.; Clet, G.; Houalla, M. Microporous Mesoporous Mater. 2005, 82, 99-104.

(31) Emeis, C. A. J. Catal. 1993, 141, 347-354.

(32) Davis, T. M.; Drews, T. O.; Ramanan, H.; He, C.; Dong, J. S.; Schnablegger, H.; Katsoulakis, M. A.; Kokkoli, E.; McCormick, A. V.; Penn, R. L.; Tsapatsis, M. Nat. Mater. 2006, 5, 400-408.

(33) Wang, F.; Richards, V. N.; Shields, S. P.; Buhro, W. E. Chem. Mater. 2014, 26, 5-21.

(34) Williamson, C. B.; Nevers, D. R.; Hanrath, T.; Robinson, R. D. J. Am. Chem. Soc. 2015, 137, 15843-15851.

(35) Jin, Y. Y.; Chen, X.; Sun, Q.; Sheng, N.; Liu, Y.; Bian, C. Q.; Chen, F.; Meng, X. J.; Xiao, F. S. Chem.-Eur. J. 2014, 20, 1761617623.
(36) Zheng, J.; Zhang, W.; Liu, Z.; Huo, Q.; Zhu, K.; Zhou, X.; Yuan, W. Microporous Mesoporous Mater. 2016, 225, 74-87.

(37) Möller, K.; Yilmaz, B.; Jacubinas, R. M.; Müller, U.; Bein, T. J. Am. Chem. Soc. 2011, 133, 5284-5295.

(38) Liu, Y.; Withers, R. L. J. Solid State Chem. 2003, 172, 431-437.

(39) Lee, S.; Jo, C.; Ryoo, R. J. Mater. Chem. A 2017, 5, 1108611093.

(40) Groen, J. C.; Peffer, L. A. A.; Pérez-Ramírez, J. Microporous Mesoporous Mater. 2003, 60, 1-17.

(41) Mériaudeau, P.; Tuan, V. A.; Nghiem, V. T.; Lai, S. Y.; Hung, L. N.; Naccache, C. J. Catal. 1997, 169, 55-66.

(42) Guo, L.; Bao, X.; Fan, Y.; Shi, G.; Liu, H.; Bai, D. J. Catal. 2012, 294, 161-170.

(43) Guo, L.; Fan, Y.; Bao, X. J.; Shi, G.; Liu, H. Y. J. Catal. 2013, 301, 162-173.

(44) Inayat, A.; Knoke, I.; Spiecker, E.; Schwieger, W. Angew. Chem., Int. Ed. 2012, 51, 1962-1965.

(45) Zhang, X. Y.; Liu, D. X.; Xu, D. D.; Asahina, S.; Cychosz, K. A.; Agrawal, K. V.; Al Wahedi, Y.; Bhan, A.; Al Hashimi, S.; Terasaki, O.; Thommes, M.; Tsapatsis, M. Science 2012, 336, 1684-1687.

(46) Zhang, S.; Chen, S.-L.; Dong, P.; Yuan, G.; Xu, K. Appl. Catal., A 2007, 332, 46-55.

(47) Fan, Y.; Lei, D.; Shi, G.; Bao, X. Catal. Today 2006, 114, 388396.

(48) Buchholz, A.; Wang, W.; Xu, M.; Arnold, A.; Hunger, M. Microporous Mesoporous Mater. 2002, 56, 267-278.

(49) Briend, M.; Peltre, M. J.; Lamy, A.; Man, P. P.; Barthomeuf, D. J. Catal. 1992, 138, 90-100.

(50) Watanabe, Y.; Koiwai, A.; Takeuchi, H.; Hyodo, S. A.; Noda, S. J. Catal. 1993, 143, 430-436.

(51) Zubowa, H.; Alsdorf, E.; Fricke, R.; Neissendorfer, F.; RichterMendau, J.; Schreier, E.; Zeigan, D.; Zibrowius, B. J. Chem. Soc., Faraday Trans. 1990, 86, 2307-2312.

(52) Martens, J. A.; Jacobs, P. A. Stud. Surf. Sci. Catal. 1994, 85, 653685.

(53) Liu, P.; Ren, J.; Sun, Y. Microporous Mesoporous Mater. 2008, 114, 365-372.

(54) Martens, J. A.; Janssens, C.; Grobet, P. J.; Beyer, H. K.; Jacobs, P. A. In Zeolites: Facts, Figures, Future: Proceedings of the 8th International Zeolite Conference, Amsterdam, July 10-14, 1989; Jacobs, P. A., van Santen, R. A., Eds.; Studies in Surface Science and Catalysis, Vol. 49; Elsevier: Amsterdam, 1989; pp 215-225.

(55) Mériaudeau, P.; Tuan, V. A.; Lefebvre, F.; Nghiem, V. T.; Naccache, C. Microporous Mesoporous Mater. 1998, 22, 435-449.

(56) Martens, J. A.; Grobet, P. J.; Jacobs, P. A. J. Catal. 1990, 126, 299-305.

(57) Zhang, L.; Huang, Y. N. J. Mater. Chem. A 2015, 3, 4522-4529.

(58) Miller, S. J.; Lacheen, H. S.; Chen, C. Y. Ind. Eng. Chem. Res. 2016, 55, 6760-6767.

(59) Pieterse, J. A. Z.; Veefkind-Reyes, S.; Seshan, K.; Domokos, L.; Lercher, J. A. J. Catal. 1999, 187, 518-520.

(60) Fan, Y.; Xiao, H.; Shi, G.; Liu, H.; Qian, Y.; Wang, T.; Gong, G.; Bao, X. J. Catal. 2011, 279, 27-35.

(61) Parry, E. P. J. Catal. 1963, 2, 371-379.

(62) Thibault-Starzyk, F.; Stan, I.; Abello, S.; Bonilla, A.; Thomas, K.; Fernandez, C.; Gilson, J. P.; Perez-Ramirez, J. J. Catal. 2009, 264, 1114.

(63) Thibault-Starzyk, F.; Vimont, A.; Gilson, J.-P. Catal. Today 2001, 70, 227-241.

(64) Alvarez, F.; Ribeiro, F. R; Perot, G.; Thomazeau, C.; Guisnet, M. J. Catal. 1996, 162, 179-189.

(65) Kondo, J. N.; Yang, S. W.; Zhu, Q. J.; Inagaki, S.; Domen, K. J. Catal. 2007, 248, 53-59.

(66) Villegas, J. I.; Kumar, N.; Heikkilä, T.; Lehto, V. P.; Salmi, T.; Murzin, D. Y. Chem. Eng. J. 2006, 120, 83-89.

(67) Groen, J. C.; Zhu, W.; Brouwer, S.; Huynink, S. J.; Kapteijn, F.; Moulijn, J. A.; Pérez-Ramírez, J. J. Am. Chem. Soc. 2007, 129, 355-360. 
(68) Saint Remi, J. C.; Lauerer, A.; Chmelik, C.; Vandendael, I.; Terryn, H.; Baron, G. V.; Denayer, J. F. M.; Karger, J. Nat. Mater. 2015, 15, 401-406.

(69) Ono, Y. Catal. Today 2003, 81, 3-16. 